\title{
Dynamical analysis of a fractional-order eco-epidemiological model with disease in prey population
}

\author{
Mahmoud Moustafa ${ }^{1 *}$ (D), Mohd Hafiz Mohd ${ }^{1}$, Ahmad Izani Ismail ${ }^{1}$ and Farah Aini Abdullah'
}

\author{
"Correspondence: \\ mahmoudmoustafa949@gmail.com \\ 'School of Mathematical Sciences, \\ Universiti Sains Malaysia, Pulau \\ Pinang, Malaysia
}

\begin{abstract}
A fractional-order eco-epidemiological model with disease in the prey population is formulated and analyzed. Mathematical analysis and numerical simulations are performed to clarify the characteristics of the proposed fractional-order model. The existence, uniqueness, non-negativity and boundedness of the solutions are proved. The local and global asymptotic stability of all equilibrium points are investigated. Finally, numerical simulations are conducted to illustrate the analytical results. The occurrence of Hopf bifurcations and transcritical bifurcations for the fractional-order eco-epidemiological model are demonstrated. It is observed that the fractional order has a stabilization effect and it may help to control the coexistence between susceptible prey, infected prey and predator populations.
\end{abstract}

Keywords: Eco-epidemiological model; Predator-prey; Fractional-order system; Stability; Bifurcations; Numerical simulation

\section{Introduction}

The dynamics of the relationship between predators and their prey are topics of considerable interest in ecology and mathematical biology. The Lotka-Volterra model is the first system that modeled the interactions between prey and its predator [1]. Studies of the dynamics of prey-predator models include [2-5]. Kermack and MacKendrick [6] proposed the classical SIR model which has drawn much attention among the scholars. Li et al. [7] studied the global stability of an SI epidemic model with feedback controls in a patchy environment. The epidemiological models consider the spread of infectious diseases related to one species is one of the major issues in mathematical biology. The modeling of infectious diseases studies including [8,9].

It is realistic to consider the impact of interacting species when epidemiological models are studied [10]. Eco-epidemiological models consider the relationships between prey and predator in which infectious diseases play a fundamental role in the dynamics of the system [11]. The eco-epidemiological model studies the spread of diseases among the prey and predator populations and one of the main aims is to control of infectious diseases. Hilker and Schmitz [12] observed that introducing disease into the Rosenzweig-

(c) The Author(s) 2020. This article is licensed under a Creative Commons Attribution 4.0 International License, which permits use sharing, adaptation, distribution and reproduction in any medium or format, as long as you give appropriate credit to the original author(s) and the source, provide a link to the Creative Commons licence, and indicate if changes were made. The images or other third party material in this article are included in the article's Creative Commons licence, unless indicated otherwise in a credit line to the material. If material is not included in the article's Creative Commons licence and your intended use is not permitted by statutory regulation or exceeds the permitted use, you will need to obtain permission directly from the copyright holder. To view a copy of this licence, visit http://creativecommons.org/licenses/by/4.0/. 
Table 1 Table of the biological description of variables and parameters

\begin{tabular}{ll}
\hline Variables \& parameters & Description \\
\hline$S$ & Susceptible prey population density. \\
$l$ & Infected prey population density. \\
$P$ & Predator population density. \\
$\hat{r}$ & Recruitment rate into prey population. \\
$\hat{\beta}$ & Transmission coefficient of disease in prey. \\
$\hat{\gamma}$ & Recovery rate of infected prey. \\
$\hat{\alpha}$ & Attack rate of predator. \\
$\hat{a}$ & Half saturation constant. \\
$\hat{c}$ & Conversion efficiency of predator. \\
$\hat{d}_{1}$ & Natural death rate of susceptible prey. \\
$\hat{d}_{2}$ & Natural death rate of infected prey. \\
$\hat{d}_{3}$ & Natural death rate of predator. \\
\hline
\end{tabular}

MacArthur model can stabilize prey-predator oscillations. Some studies have been carried out on eco-epidemiological models with disease either in prey [13-16] or in predator [17-20] or in both populations [21-23]. The infectious diseases among prey and predator populations are disorders caused by bacteria or virus [24].

In [11] the authors considered a three-species eco-epidemiological model with Holling type II functional response of a predator. It is assumed that population can be divided into susceptible prey, infected prey and predator; disease spreads only among the preys; infected prey can recover and predator eats only infected prey.

Following $[10,11]$ a new version of an eco-epidemiological model is formulated by incorporating a predator's attack rate and half saturation constant as follows:

$$
\begin{aligned}
& \frac{d S}{d t}=\hat{r}-\hat{\beta} S I-\hat{d}_{1} S+\hat{\gamma} I, \\
& \frac{d I}{d t}=\hat{\beta} S I-\frac{\hat{\alpha} I P}{1+\hat{a} I}-\hat{d}_{2} I-\hat{\gamma} I, \\
& \frac{d P}{d t}=\frac{\hat{c} \hat{\alpha} I P}{1+\hat{a} I}-\hat{d}_{3} P .
\end{aligned}
$$

All the parameters are assumed to be positive. The variables and parameters of system (1) are presented in Table 1.

Fractional-order differential equations can be considered as generalization of ordinary differential equations to an arbitrary (non-integer) order and have been successfully applied in engineering, chemistry and mathematical biology $[25,26]$. The fractional-order derivative is a non-local operator in the sense that the system at present states depends on the recent past states [27].

The fractional-order differential equations exhibit richer dynamical behavior and this is because it incorporates the memory effect in the model [28]. Zhao and Luo [29] proposed a definition of general fractional derivatives to describe the dynamics with memory effects. Bolton et al. [30] conclude that the fractional-order Gompertz growth model is more realistic as regards a experimental dataset than the integer-order Gompertz model. Therefore, the use of fractional-order differential equations may improve the modeling of biological phenomena. Hence, the dynamics of the relations between predators and their prey can be more accurately described by fractional-order systems [31, 32]. Detailed background of the fractional-order differential equations can be found in [33-41]. Some 
previous studies indicate that the fractional-order system cannot have a periodic solution $[42,43]$. But the fractional derivative converges to periodic signals if it is defined on the whole real line [44, 45]. This paper will be focused on the final state of the trajectory [44]. Hence, the limit cycle attracts all nearby positive-valued trajectories (asymptotically stable limit cycle) [44].

In $[33,34]$ a kind of fractional order eco-epidemiological model with disease in the prey population was proposed and some issues related to theoretical and numerical analyses were investigated. However, the governing systems proposed in [33, 34] are different from our fractional-order model (3). In this paper, we consider the following fractional-order eco-epidemiological model incorporating a predator's attack rate and half saturation constant:

$$
\begin{aligned}
& { }^{c} D^{q} S(t)=\hat{r}^{q}-\hat{\beta}^{q} S I-\hat{d}_{1}^{q} S+\hat{\gamma}^{q} I, \\
& { }^{c} D^{q} I(t)=\hat{\beta}^{q} S I-\frac{\hat{\alpha}^{q} I P}{1+\hat{a} I}-\hat{d}_{2}^{q} I-\hat{\gamma}^{q} I, \\
& { }^{c} D^{q} P(t)=\frac{\hat{c} \hat{\alpha}^{q} I P}{1+\hat{a} I}-\hat{d}_{3}^{q} P,
\end{aligned}
$$

with initial conditions

$$
S(0)=S_{0} \geq 0, \quad I(0)=I_{0} \geq 0, \quad P(0)=P_{0} \geq 0,
$$

where $0<q<1$ and ${ }^{c} D^{q}$ is the standard Caputo differentiation. The parameters in the fractional-order eco-epidemiological model (2) are assumed to be positive and are described in Table 1. The Caputo fractional derivative of order $q$ is defined as [26]

$$
{ }^{c} D^{q} f(t)=\frac{1}{\Gamma(n-q)} \int_{0}^{t}(t-s)^{n-q-1} f^{(n)}(s) d s, \quad n-1<q<n, n \in \mathbb{N} .
$$

However, it is important to note that the modified parameters, such as $\hat{r}^{q}$ depending on the fractional order $(q)$ and that the units of each differential equation terms are different $[46,47]$. Also, the fractional derivative equations are formulated not with respect to the physical time but with respect to an intrinsic time variable that depends on $q$ [48]. For simplification, this system can be redefined with the new parameters as follows [49]:

$$
\begin{array}{ll}
\hat{r}^{q}=r, \quad \hat{\beta}^{q}=\beta, \quad \hat{d}_{1}^{q}=d_{1}, \quad \hat{d}_{2}^{q}=d_{2}, \quad \hat{d}_{3}^{q}=d_{3}, \quad \hat{c}=c, \\
\hat{\gamma}^{q}=\gamma, \quad \hat{\alpha}^{q}=\alpha, \quad \hat{a}=a .
\end{array}
$$

Then, the system (2) becomes as follows:

$$
\begin{aligned}
& { }^{c} D^{q} S(t)=r-\beta S I-d_{1} S+\gamma I, \\
& { }^{c} D^{q} I(t)=\beta S I-\frac{\alpha I P}{1+a I}-d_{2} I-\gamma I, \\
& { }^{c} D^{q} P(t)=\frac{c \alpha I P}{1+a I}-d_{3} P .
\end{aligned}
$$

So far as we are aware, no scholar has investigated the dynamics of the fractional-order eco-epidemiological model (3). Therefore, in this paper, a fractional order prey-predator 
model with disease in the prey population is investigated. The mathematical analysis and numerical simulations are performed to clarify the characteristics of our fractional-order model (3). The aim of this study is to proposed and analyzed a fractional-order ecoepidemiological model incorporating predator's attack rate $(\alpha)$ and half saturation constant $(a)$ with infection in prey population. Also, we observe the effects of the prey's disease $(\beta)$, predator's attack rate $(\alpha)$, half saturation constant $(a)$, infected prey's death rate $\left(d_{2}\right)$ and fractional order $(q)$ in the dynamics of the fractional-order eco-epidemiological model (3). The contributions of this paper are to prove existence, uniqueness, non-negativity, and boundedness of the solutions for model (3). The paper also investigates the local and global asymptotic stability of all equilibrium points of the fractional order model (3) by using Matignon's condition and constructing suitable Lyapunov functions, respectively. Numerical simulations conducted indicate a rich dynamical behavior of the fractional order model (3) at the equilibrium points, which is in agreement with the theoretical analysis.

The paper is organized as follows. In the next section, the analysis of the fractionalorder eco-epidemiological model (3) is presented. In Sect. 3, the numerical simulations of the fractional-order eco-epidemiological model (3) are provided to verify the theoretical results. Finally, Sect. 4 concludes the study with a brief discussion.

\section{Mathematical analysis}

The mathematical analysis of the fractional-order eco-epidemiological model (3) is presented in this section.

\subsection{Existence and uniqueness}

The existence and uniqueness of the solutions of the fractional-order system (3) are studied in the region $\Omega \times(0, T]$ where

$$
\Omega=\left\{(S, I, P) \in \mathbb{R}^{3}: \max (|S|,|I|,|P|) \leq \psi\right\} .
$$

Theorem 1 For each $X_{0}=\left(S_{0}, I_{0}, P_{0}\right) \in \Omega$, there exists a unique solution $X(t) \in \Omega$ of the fractional-order system (3) with initial condition $X_{0}$, which is defined for all $t \geq 0$.

Proof The approach used by [50] is utilized. For $X, \bar{X} \in \Omega$, one can consider a mapping $M(X)=\left(M_{1}(X), M_{2}(X), M_{3}(X)\right)$ where

$$
\begin{aligned}
& M_{1}(X)=r-\beta S I-d_{1} S+\gamma I, \\
& M_{2}(X)=\beta S I-\frac{\alpha I P}{1+a I}-d_{2} I-\gamma I, \\
& M_{3}(X)=\frac{c \alpha I P}{1+a I}-d_{3} P .
\end{aligned}
$$

It follows from (4) that

$$
\begin{aligned}
& \|M(X)-M(\bar{X})\| \\
& \quad=\left|M_{1}(X)-M_{1}(\bar{X})\right|+\left|M_{2}(X)-M_{2}(\bar{X})\right|+\left|M_{3}(X)-M_{3}(\bar{X})\right| \\
& \quad=\left|r-\beta S I-d_{1} S+\gamma I-r+\beta \bar{S} \bar{I}+d_{1} \bar{S}-\gamma \bar{I}\right|
\end{aligned}
$$




$$
\begin{aligned}
& +\left|\beta S I-\frac{\alpha I P}{1+a I}-d_{2} I-\gamma I-\beta \bar{S} \bar{I}+\frac{\alpha \bar{I} \bar{P}}{1+a \bar{I}}+d_{2} \bar{I}+\gamma \bar{I}\right| \\
& +\left|\frac{c \alpha I P}{1+a I}-d_{3} P-\frac{c \alpha \bar{I} \bar{P}}{1+a \bar{I}}+d_{3} \bar{P}\right| \\
= & -\beta(S I-\bar{S} \bar{I})-d_{1}(S-\bar{S})+\gamma(I-\bar{I}) \mid \\
& +\left|-\frac{\alpha(I P+a \bar{I} \bar{P}-\bar{I} \bar{P}-a I \bar{I} \bar{P})}{(1+a I)(1+a \bar{I})}+\beta(S I-\bar{S} \bar{I})-d_{2}(I-\bar{I})-\gamma(I-\bar{I})\right| \\
& +\left|\frac{c \alpha(I P+a I \bar{I} P-\bar{I} \bar{P}-a \bar{I} \bar{P})}{(1+a I)(1+a \bar{I})}-d_{3}(P-\bar{P})\right| \\
\leq & \beta \psi|S-\bar{S}|+\beta \psi|I-\bar{I}|+d_{1}|S-\bar{S}|+\gamma|I-\bar{I}| \\
& +\frac{\alpha(1+c)|P-\bar{P}|}{a}+\beta \psi|S-\bar{S}|+\beta \psi|I-\bar{I}|+d_{2}|I-\bar{I}|+\gamma|I-\bar{I}| \\
& +d_{3}|P-\bar{P}|+\alpha(1+c) \psi|I-\bar{I}|+\alpha(1+c) \psi|P-\bar{P}| \\
\leq & \left(2 \beta \psi+d_{1}\right)|S-\bar{S}| \\
& +\left(2 \beta \psi+2 \gamma+d_{2}+\alpha(1+c) \psi\right)|I-\bar{I}| \\
& +\left(\frac{\alpha(1+c)}{a}+d_{3}+\alpha(1+c) \psi\right)|P-\bar{P}| \\
\leq & G\|X-\bar{X}\|,
\end{aligned}
$$

where

$$
G=\max \left\{2 \beta \psi+d_{1}, 2 \beta \psi+2 \gamma+d_{2}+\alpha(1+c) \psi, \frac{\alpha(1+c)}{a}+d_{3}+\alpha(1+c) \psi\right\}
$$

Thus, $M(X)$ satisfies the Lipschitz condition which proves the existence and uniqueness of the solutions of the fractional-order eco-epidemiological model (3).

\subsection{Non-negativity and boundedness}

Now, the non-negativity of the solutions of the fractional-order system (3) is studied. From (3), one has

$$
\begin{aligned}
& \left.{ }^{c} D^{q} S(t)\right|_{S=0}=r+\gamma I \geq 0, \\
& \left.{ }^{c} D^{q} I(t)\right|_{I=0}=0, \\
& \left.{ }^{c} D^{q} P(t)\right|_{P=0}=0 .
\end{aligned}
$$

According to Lemmas 5 and 6 in [51], one can deduce that the solutions of the fractionalorder system (3) are non-negative.

Next, the boundedness of the solutions of the fractional-order system (3) is given.

Theorem 2 The solutions of the fractional-order system (3) which start in $\mathbb{R}_{+}^{3}$ are uniformly bounded. 
Proof The approach of [50] is adopted. Let us consider the function $H(t)=S(t)+I(t)+$ $\frac{1}{c} P(t)$. Then

$$
\begin{aligned}
{ }^{c} D^{q} H(t) & ={ }^{c} D^{q} S(t)+{ }^{c} D^{q} I(t)+\frac{1}{c}{ }^{c} D^{q} P(t) \\
& =r-d_{1} S-d_{2} I-\frac{d_{3}}{c} P .
\end{aligned}
$$

Hence, for each $\lambda>0$,

$$
\begin{aligned}
{ }^{c} D^{q} H(t)+\lambda H(t) & =r-d_{1} S-d_{2} I-\frac{d_{3}}{c} P+\lambda S+\lambda I+\frac{\lambda}{c} P \\
& =r+\left(\lambda-d_{1}\right) S+\left(\lambda-d_{2}\right) I+\frac{1}{c}\left(\lambda-d_{3}\right) P .
\end{aligned}
$$

One can choose $\lambda<\min \left\{d_{1}, d_{2}, d_{3}\right\}$. Thus

$$
{ }^{c} D^{q} H(t)+\lambda H(t) \leq r .
$$

According to Lemma 9 in [52], it follows that

$$
0 \leq H(t) \leq H(0) E_{q}\left(-\lambda(t)^{q}\right)+r(t)^{q} E_{q, q+1}\left(-\lambda(t)^{q}\right),
$$

where $E_{q}$ is the well-known Mittag-Leffler function. By Lemma 5 and Corollary 6 in [52], one gets the following expression:

$$
0 \leq H(t) \leq \frac{r}{\lambda}, \quad \text { as } t \rightarrow \infty
$$

Therefore, the solutions of fractional-order eco-epidemiological model (3) starting in $\mathbb{R}_{+}^{3}$ are uniformly bounded in the region $W$, where

$$
W=\left\{(S, I, P) \in R_{+}^{3}: H(t) \leq \frac{r}{\lambda}+\epsilon, \epsilon>0\right\} .
$$

\subsection{Equilibrium points and stability}

In this section, we investigate the stability of equilibrium points of the fractional-order system (3). The fractional-order system (3) has the following equilibrium points:

1. The axial equilibrium point $E_{1}\left(\frac{r}{d_{1}}, 0,0\right)$, which always exists.

2. The predator-extinction equilibrium point $E_{2}\left(\frac{\gamma+d_{2}}{\beta}, \frac{d_{1}\left(\gamma+d_{2}\right)\left(\Re_{0}-1\right)}{\beta d_{2}}, 0\right)$, which exists if $\Re_{0}>1$, where $\Re_{0}=\frac{r \beta}{d_{1}\left(\gamma+d_{2}\right)}$ is the basic reproduction number obtained by using the next generation method [53].

3. The interior equilibrium point $E_{3}\left(S_{3}, I_{3}, P_{3}\right)$ where

$$
\begin{aligned}
& S_{3}=\frac{r\left(c \alpha-a d_{3}\right)+\gamma d_{3}}{d_{1}\left(c \alpha-a d_{3}\right)+\beta d_{3}}, \quad I_{3}=\frac{d_{3}}{c \alpha-a d_{3}} \quad \text { and } \\
& P_{3}=\frac{c d_{1}\left(\gamma+d_{2}\right)\left(\Re_{0}-1-\frac{\beta d_{2} d_{3}}{d_{1}\left(\gamma+d_{2}\right)\left(c \alpha-a d_{3}\right)}\right)}{d_{1}\left(c \alpha-a d_{3}\right)+\beta d_{3}} .
\end{aligned}
$$

The interior equilibrium point $E_{3}$ exists if $\alpha>\frac{a d_{3}}{c}$ and $\Re_{0}>1+\frac{\beta d_{2} d_{3}}{d_{1}\left(\gamma+d_{2}\right)\left(c \alpha-a d_{3}\right)}$ which is equivalent to $\alpha>\frac{\left(\beta\left(a r+d_{2}\right)-a d_{1}\left(\gamma+d_{2}\right)\right) d_{3}}{c\left(r \beta-d_{1}\left(\gamma+d_{2}\right)\right)}$. 
Thus, it is observed that the existence of interior equilibrium point $E_{3}$ depends on the threshold value of the predator's attack rate $(\alpha)$ and the basic reproduction number $\left(\Re_{0}\right)$, respectively. So, the attack rate of predator and basic reproduction number play some crucial roles in determining the dynamics of the fractional-order system (3). Next, we will discuss the stability of the equilibrium points of system (3). The Jacobian matrix of system (3) is given by

$$
J(S, I, P)=\left(\begin{array}{ccc}
-\left(I \beta+d_{1}\right) & \gamma-\beta S & 0 \\
\beta I & \frac{(1+a I)^{2}(\beta S-\gamma)-\alpha P}{(1+a I)^{2}}-d_{2} & -\frac{\alpha I}{1+a I} \\
0 & \frac{c \alpha P}{(1+a I)^{2}} & \frac{c \alpha I}{1+a I}-d_{3}
\end{array}\right) .
$$

Utilizing the Jacobian matrix (6) and the Matignon condition [32,54], the local stability of the equilibrium points of the fractional-order system (3) is investigated.

Theorem 3 The axial equilibrium point $E_{1}$ of the fractional-order system (3) is locally asymptotically stable if $\Re_{0}<1$ and unstable saddle point if $\Re_{0}>1$.

Proof The Jacobian matrix (6) around the axial equilibrium point $E_{1}$ is as follows:

$$
J\left(E_{1}\right)=\left(\begin{array}{ccc}
-d_{1} & \gamma-\frac{r \beta}{d_{1}} & 0 \\
0 & \frac{r \beta}{d_{1}}-\left(\gamma+d_{2}\right) & 0 \\
0 & 0 & -d_{3}
\end{array}\right) .
$$

The eigenvalues of the Jacobian matrix around the axial equilibrium point $E_{1}$ are $\mu_{1}=-d_{1}$, $\mu_{2}=-d_{3}$ and $\mu_{3}=\frac{r \beta-d_{1}\left(\gamma+d_{2}\right)}{d_{1}}$. By using Matignon's condition [32, 54], it can be observed that $\left|\arg \left(\mu_{1,2}\right)\right|=\pi>\frac{q \pi}{2}$. If $\Re_{0}<1$ then $\left|\arg \left(\mu_{3}\right)\right|=\pi>\frac{q \pi}{2}$ for all $0<q<1$. Thus, the axial equilibrium point $E_{1}$ is locally asymptotically stable if $\Re_{0}<1$, which is equivalent to $\gamma>$ $\frac{r \beta-d_{1} d_{2}}{d_{1}}$. It is observed that the axial equilibrium point $E_{1}$ is locally asymptotically stable when the predator-extinction equilibrium point $E_{2}$ and interior equilibrium point $E_{3}$ do not exist.

Now, the stability of the predator-extinction equilibrium point $E_{2}\left(S_{2}, I_{2}, 0\right)$ is discussed.

Theorem 4 If $\Re_{0}<1+\frac{\beta d_{2} d_{3}}{d_{1}\left(\gamma+d_{2}\right)\left(c \alpha-a d_{3}\right)}$, then the predator-extinction equilibrium point $E_{2}$ of the fractional-order model (3) is locally asymptotically stable.

Proof The Jacobian matrix (6) evaluated at $E_{2}$ is given by

$$
J\left(E_{2}\right)=\left(\begin{array}{ccc}
-\left(d_{1}+\beta I_{2}\right) & -d_{2} & 0 \\
\beta I_{2} & 0 & -\frac{\alpha I_{2}}{1+a I_{2}} \\
0 & 0 & \frac{c \alpha I_{2}}{1+a I_{2}}-d_{3}
\end{array}\right) .
$$

The eigenvalues of the Jacobian matrix around $E_{2}$ are the roots of the following equation:

$$
\left(\frac{c \alpha I_{2}}{1+a I_{2}}-d_{3}-\mu\right)\left(\mu^{2}+\mu\left(\beta I_{2}+d_{1}\right)+\beta d_{2} I_{2}\right)=0
$$


The characteristic equation (7) has the following eigenvalues:

$$
\mu_{1}=\frac{c \alpha I_{2}}{1+a I_{2}}-d_{3} \quad \text { and } \quad \mu_{2,3}=\frac{1}{2}\left(-\left(\beta I_{2}+d_{1}\right) \pm \sqrt{\left(\beta I_{2}+d_{1}\right)^{2}-4 \beta d_{2} I_{2}}\right) \text {. }
$$

The eigenvalues $\mu_{2,3}$ have negative real parts, then $\left|\arg \left(\mu_{2,3}\right)\right|=\pi>\frac{q \pi}{2}$. If $\Re_{0}<1+$ $\frac{\beta d_{2} d_{3}}{d_{1}\left(\gamma+d_{2}\right)\left(c \alpha-a d_{3}\right)}$ then $\left|\arg \left(\mu_{1}\right)\right|=\pi>\frac{q \pi}{2}$ for all $0<q<1$. Thus, in accordance with Matignon's condition [32, 54], the predator-extinction equilibrium point $E_{2}$ is locally asymptotically stable if $\Re_{0}<1+\frac{\beta d_{2} d_{3}}{d_{1}\left(\gamma+d_{2}\right)\left(c \alpha-a d_{3}\right)}$, which is equivalent to the attack rate of predator population $(\alpha)$ being less than $\frac{\left(\beta\left(a r+d_{2}\right)-a d_{1}\left(\gamma+d_{2}\right)\right) d_{3}}{c\left(r \beta-d_{1}\left(\gamma+d_{2}\right)\right)}$.

Finally, the stability of interior equilibrium point $E_{3}\left(S_{3}, I_{3}, P_{3}\right)$ is investigated. The Jacobian matrix of system (3) around $E_{3}$ is given by

$$
J\left(E_{3}\right)=\left(\begin{array}{ccc}
-\left(\beta I_{3}+d_{1}\right) & \gamma-\beta S_{3} & 0 \\
\beta I_{3} & \frac{\alpha P_{3}}{\left(1+a I_{3}\right)}\left(1-\frac{1}{1+a I_{3}}\right) & -\frac{\alpha I_{3}}{1+a I_{3}} \\
0 & \frac{c \alpha P_{3}}{\left(1+a I_{3}\right)^{2}} & 0
\end{array}\right) .
$$

The eigenvalues of $J\left(E_{3}\right)$ are the roots of the following equation:

$$
F(\mu)=\mu^{3}+K_{1} \mu^{2}+K_{2} \mu+K_{3}=0
$$

where

$$
\begin{aligned}
& K_{1}=d_{1}+I_{3}\left(\beta-\frac{a \alpha P_{3}}{\left(1+a I_{3}\right)^{2}}\right), \\
& K_{2}=\frac{I_{3}\left(1-\beta \gamma\left(+a I_{3}\right)^{3}-\alpha\left(a\left(1+a I_{3}\right)\left(d_{1}+\beta I_{3}\right)-c \alpha\right) P_{3}+\beta^{2}\left(1+a I_{3}\right)^{3} S_{3}\right.}{\left(1+a I_{3}\right)^{3}}, \\
& K_{3}=\frac{c \alpha^{2}\left(\beta I_{3}+d_{1}\right) I_{3} P_{3}}{\left(1+a I_{3}\right)^{3}} .
\end{aligned}
$$

According to $[55,56]$, the local stability of the interior equilibrium point $E_{3}$ is determined by the following proposition.

Proposition 5 The discriminant $D(V)$ of the cubic polynomial (8) is as follows:

$$
D(V)=18 K_{1} K_{2} K_{3}+\left(K_{1} K_{2}\right)^{2}-4 K_{3} K_{1}^{3}-4 K_{2}^{3}-27 K_{3}^{2} .
$$

Then the conditions of local stability of the interior equilibrium point $E_{3}$ are given as follows:

(i) If $D(V)>0, K_{1}>0, K_{3}>0$ and $K_{1} K_{2}>K_{3}$, then $E_{3}$ is locally asymptotically stable for $0<q<1$.

(ii) If $D(V)<0, K_{1}>0, K_{2}>0, K_{1} K_{2}=K_{3}$ and $0<q<1$, then $E_{3}$ is locally asymptotically stable.

\subsection{Global stability}

The global asymptotic stability of the axial equilibrium point $E_{1}$, predator-extinction equilibrium point $E_{2}$ and the interior equilibrium point $E_{3}$ of the fractional-order system (3) is now investigated. 
Theorem 6 If $\Re_{0}<1$, then the axial equilibrium point $E_{1}$ of the fractional-order system (3) is globally asymptotically stable.

Proof We consider the following positive definite Lyapunov function:

$$
V(S, I, P)=\left(S-\frac{r}{d_{1}}-\frac{r}{d_{1}} \ln \frac{d_{1} S}{r}\right)+I+\frac{1}{c} P .
$$

By calculating the $q$-order derivative of $V(S, I, P)$ along the solution of system (3) and utilizing Lemma 3.1 in [57], one has

$$
\begin{aligned}
{ }^{c} D^{q} V(S, I, P) \leq & \left(\frac{d_{1} S-r}{d_{1} S}\right){ }^{c} D^{q} S(t)+{ }^{c} D^{q} I(t)+\frac{1}{c}{ }^{c} D^{q} P(t) \\
\leq & \frac{d_{1} S-r}{d_{1} S}\left(r-\beta S I-d_{1} S+\gamma I\right) \\
& +\beta S I-d_{2} I-\gamma I-\frac{d_{3}}{c} P \\
\leq & \frac{d_{1} S-r}{d_{1} S}\left(-\left(S d_{1}+r\right)-(\beta S-\gamma) I\right) \\
& +\beta S I-d_{2} I-\gamma I-\frac{d_{3}}{c} P \\
\leq & \frac{-\left(d_{1} S-r\right)^{2}}{d_{1} S}-\frac{\left(S d_{1}-r\right)(\beta S-\gamma) I}{d_{1} S} \\
& +\beta S I-d_{2} I-\gamma I-\frac{d_{3}}{c} P \\
\leq & \frac{-\left(d_{1} S-r\right)^{2}}{d_{1} S}+\frac{r \beta I}{d_{1}}-\frac{\gamma r I}{d_{1} S}-d_{2} I-\frac{d_{3}}{c} P \\
\leq & \frac{r \beta I}{d_{1}}-\frac{\gamma r I}{d_{1} S}-d_{2} I \\
\leq & \left(\frac{\beta r}{d_{1}}-\gamma-d_{2}\right) I .
\end{aligned}
$$

Thus, ${ }^{c} D^{q} V(S, I, P) \leq 0$ when $\frac{\beta r}{d_{1}}<\gamma+d_{2}$ which is equivalent to $\Re_{0}<1$. By Lemma 4.6 in [58], it is proof that the axial equilibrium point $E_{1}$ is globally asymptotically stable.

Theorem 7 If $\Re_{0}<1+\frac{\beta d_{2} d_{3}}{d_{1}\left(\gamma+d_{2}\right)\left(c \alpha-a d_{3}\right)}$, then the predator-extinction equilibrium point $E_{2}$ is globally asymptotically stable.

Proof To study the globally asymptotically stability of $E_{2}$ the following positive definite Lyapunov function is considered:

$$
V(S, I, P)=L\left(S-S_{2}-S_{2} \ln \frac{S}{S_{2}}\right)+\left(I-I_{2}-I_{2} \ln \frac{I}{I_{2}}\right)+\frac{1}{c} P .
$$

The $q$-order derivative of $V(S, I, P)$ is calculating along the solution of the fractional-order eco-epidemiological model (3) and by applying Lemma 3.1 in [57], we get

$$
{ }^{c} D^{q} V(S, I, P) \leq L\left(\frac{S-S_{2}}{S}\right)^{c} D^{q} S(t)+\left(\frac{I-I_{2}}{I}\right){ }^{c} D^{q} I(t)+\frac{1}{c}{ }^{c} D^{q} P(t)
$$




$$
\begin{aligned}
\leq & L\left(\frac{S-S_{2}}{S}\right)\left(r-\beta S I-d_{1} S+\gamma I\right) \\
& +\left(I-I_{2}\right)\left(\beta S-\frac{\alpha P}{1+a I}-d_{2}-\gamma\right)+P\left(\frac{\alpha I}{1+a I}-\frac{d_{3}}{c}\right) \\
\leq & L\left(\frac{S-S_{2}}{S}\right)\left(\beta S_{2} I_{2}+d_{1} S_{2}-\gamma I_{2}-\beta S I-d_{1} S+\gamma I\right) \\
& +\left(I-I_{2}\right)\left(\beta S-\frac{\alpha P}{1+a I}-\beta S_{2}\right)+\frac{\alpha I P}{1+a I}-\frac{d_{3}}{c} P \\
\leq & L\left(\frac{S-S_{2}}{S}\right)\left(-\beta I_{2}\left(S-S_{2}\right)-\beta S\left(I-I_{2}\right)-d_{1}\left(S-S_{2}\right)+\gamma\left(I-I_{2}\right)\right) \\
& +\left(I-I_{2}\right)\left(\beta\left(S-S_{2}\right)-\frac{\alpha P}{1+a I}\right)+\frac{\alpha I P}{1+a I}-\frac{d_{3}}{c} P,
\end{aligned}
$$

since $\frac{\gamma L}{S}\left(S-S_{2}\right)\left(I-I_{2}\right)=\frac{-\gamma L}{S S_{2}}\left(I-I_{2}\right)\left(S-S_{2}\right)^{2}+\frac{\gamma L}{S_{2}}\left(S-S_{2}\right)\left(I-I_{2}\right)$,

$$
\begin{aligned}
{ }^{c} D^{q} V(S, I, P) \leq & \left(\frac{\gamma L I_{2}}{S S_{2}}\left(S-S_{2}\right)^{2}-\frac{\left(\beta I_{2}+d_{1}\right) L}{S}\left(S-S_{2}\right)^{2}\right) \\
& -\frac{\gamma L I}{S S_{2}}\left(S-S_{2}\right)^{2}+\left(\beta-\beta L+\frac{\gamma L}{S_{2}}\right)\left(S-S_{2}\right)\left(I-I_{2}\right) \\
& +\left(\frac{\alpha I_{2}}{1+a I}-\frac{d_{3}}{c}\right) P \\
\leq & -\frac{r L}{S S_{2}}\left(S-S_{2}\right)^{2}+\frac{1}{S_{2}}\left(\beta S_{2}-\beta L S_{2}+\gamma L\right)\left(S-S_{2}\right)\left(I-I_{2}\right) \\
& +\left(\alpha I_{2}-\frac{d_{3}}{c}\right) P .
\end{aligned}
$$

Suppose $L=\frac{\beta S_{2}}{\beta S_{2}-\gamma}=1+\frac{\gamma}{d_{2}}>0$. Hence, ${ }^{c} D^{q} V(S, I, P) \leq 0$, when $\alpha I_{2}-\frac{d_{3}}{c}<0$, which equivalent to

$$
\Re_{0}<1+\frac{\beta d_{2} d_{3}}{d_{1}\left(\gamma+d_{2}\right)\left(c \alpha-a d_{3}\right)} .
$$

Hence the theorem is proved.

Theorem 8 The interior equilibrium point $E_{3}$ of the fractional-order system (3) is globally asymptotically stable if $\alpha a P_{3} \theta S_{3}\left(\theta^{2}-2 \xi I_{3}+I_{3}^{2}\right)<\gamma \xi M\left(1+a I_{3}\right)(1+a \xi)\left(\xi^{2}-2 \theta S_{3}+S_{3}^{2}\right)$.

Proof The following positive definite Lyapunov function is considered to investigate the global asymptotic stability of the interior equilibrium point $E_{3}$ :

$$
V(S, I, P)=M\left(S-S_{3}-S_{3} \ln \frac{S}{S_{3}}\right)+\left(I-I_{3}-I_{3} \ln \frac{I}{I_{3}}\right)+\left(\frac{1+a I_{3}}{c}\right)\left(P-P_{3}-P_{3} \ln \frac{P}{P_{3}}\right) .
$$

We calculate the time derivative of $V(S, I, P)$ along the solution of model (3) and by applying Lemma 3.1 in [57], one has 


$$
\begin{aligned}
& \leq M\left(\frac{S-S_{3}}{S}\right){ }^{c} D^{q} S(t)+\left(\frac{I-I_{3}}{I}\right){ }^{c} D^{q} I(t)+\left(\frac{1+a I_{3}}{c}\right)\left(\frac{P-P_{3}}{P}\right){ }^{c} D^{q} P(t) \\
& \leq M\left(\frac{S-S_{3}}{S}\right)\left(r-\beta S I-d_{1} S+\gamma I\right) \\
& +\left(I-I_{3}\right)\left(\beta S-\frac{\alpha P}{1+a I}-\left(d_{2}+\gamma\right)\right)+\left(1+a I_{3}\right)\left(P-P_{3}\right)\left(\frac{\alpha I}{1+a I}-\frac{d_{3}}{c}\right) \\
& \leq M\left(\frac{S-S_{3}}{S}\right)\left(-d_{1}\left(S-S_{3}\right)+\gamma\left(I-I_{3}\right)-\beta I_{3}\left(S-S_{3}\right)-\beta S\left(I-I_{3}\right)\right) \\
& +\left(I-I_{3}\right)\left(\beta S-\frac{\alpha P}{1+a I}-\beta S_{3}+\frac{\alpha P_{3}}{1+a I_{3}}\right) \\
& +\left(1+a I_{3}\right)\left(P-P_{3}\right) \alpha\left(\frac{I}{1+a I}-\frac{I_{3}}{1+a I_{3}}\right) \\
& \leq-\frac{\left(d_{1}+\beta I_{3}\right) M}{S}\left(S-S_{3}\right)^{2}+\frac{\gamma M}{S}\left(S-S_{3}\right)\left(I-I_{3}\right) \\
& -\beta M\left(S-S_{3}\right)\left(I-I_{3}\right)+\left(I-I_{3}\right)\left(\beta S-\frac{\alpha P}{1+a I}-\beta S_{3}+\frac{\alpha P_{3}}{1+a I_{3}}\right) \\
& +\left(1+a I_{3}\right)\left(P-P_{3}\right) \alpha\left(\frac{I}{1+a I}-\frac{I_{3}}{1+a I_{3}}\right) \\
& \leq-\frac{\left(d_{1}+\beta I_{3}\right) M}{S}\left(S-S_{3}\right)^{2}+\frac{\gamma M}{S_{3}}\left(S-S_{3}\right)\left(I-I_{3}\right) \\
& -\frac{\gamma M}{S S_{3}}\left(S-S_{3}\right)^{2}\left(I-I_{3}\right)-\beta M\left(S-S_{3}\right)\left(I-I_{3}\right) \\
& +\left(I-I_{3}\right)\left(\beta S-\frac{\alpha P}{1+a I}-\beta S_{3}+\frac{\alpha P_{3}}{1+a I_{3}}\right) \\
& +\left(1+a I_{3}\right)\left(P-P_{3}\right) \alpha\left(\frac{I}{1+a I}-\frac{I_{3}}{1+a I_{3}}\right) \\
& \leq \frac{M\left(S-S_{3}\right)^{2}}{S S_{3}}\left(\gamma I_{3}-\beta I_{3} S_{3}-d_{1} S_{3}\right) \\
& +\frac{1}{S_{3}}\left(S-S_{3}\right)\left(I-I_{3}\right)\left(\gamma M-\beta M S_{3}+\beta S_{3}\right) \\
& +\frac{\alpha P_{3}\left(I-I_{3}\right)}{1+a I_{3}}-\frac{\alpha P_{3}\left(I-I_{3}\right)}{1+a I}-\frac{\gamma M I}{S S_{3}}\left(S-S_{3}\right)^{2} \\
& \leq-\frac{r M\left(S-S_{3}\right)^{2}}{S S_{3}}+\frac{1}{S_{3}}\left(S-S_{3}\right)\left(I-I_{3}\right)\left(\gamma M-\beta M S_{3}+\beta S_{3}\right) \\
& +\frac{\alpha a P_{3}\left(I^{2}-2 I I_{3}+I_{3}^{2}\right)}{\left(1+a I_{3}\right)(1+a I)}-\frac{\gamma M I\left(S^{2}-2 S S_{3}+S_{3}^{2}\right)}{S S_{3}} \\
& \leq-\frac{r M\left(S-S_{3}\right)^{2}}{S S_{3}}+\frac{1}{S_{3}}\left(S-S_{3}\right)\left(I-I_{3}\right)\left(\gamma M-\beta M S_{3}+\beta S_{3}\right) \\
& +\frac{\alpha a P_{3}\left(\theta^{2}-2 \xi I_{3}+I_{3}^{2}\right)}{\left(1+a I_{3}\right)(1+a \xi)}-\frac{\gamma M \xi\left(\xi^{2}-2 \theta S_{3}+S_{3}^{2}\right)}{\theta S_{3}} .
\end{aligned}
$$

Suppose $M=\frac{\beta S_{3}}{\beta S_{3}-\gamma}=\frac{\beta S_{3}\left(1+a I_{3}\right)}{\alpha P_{3}+d_{2}\left(1+a I_{3}\right)}>0$ and $\xi<S, I<\theta$. Thus, ${ }^{c} D^{q} V(S, I, P) \leq 0$, when $\alpha a P_{3} \theta S_{3}\left(\theta^{2}-2 \xi I_{3}+I_{3}^{2}\right)<\gamma \xi M\left(1+a I_{3}\right)(1+a \xi)\left(\xi^{2}-2 \theta S_{3}+S_{3}^{2}\right)$. By Lemma 4.6 in [58], it is proof that the interior equilibrium point $E_{3}$ is globally asymptotically stable. 

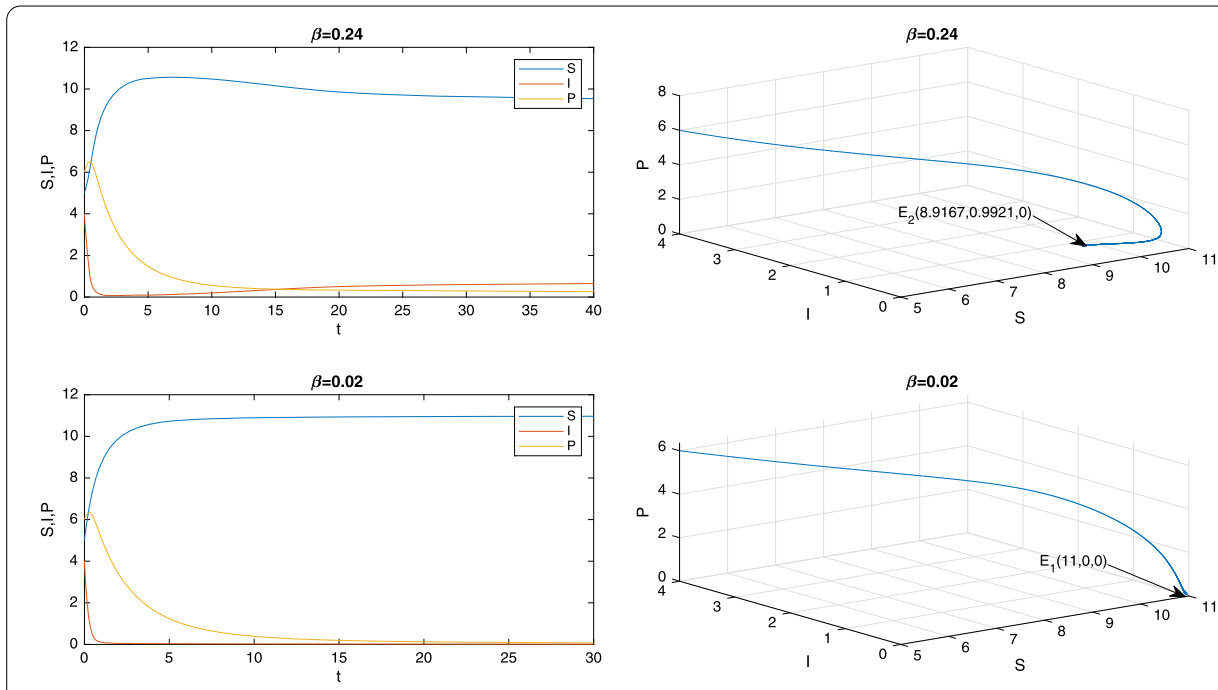

Figure 1 Time series and phase diagram of the predator-extinction equilibrium point $E_{2}(8.9167,0.9921,0)$ and axial equilibrium point $E_{1}(11,0,0)$ with different values of $\beta$ and $q=0.9$

\section{Numerical simulations}

In this section, numerical simulations of the fractional-order eco-epidemiological model (3) are conducted to illustrate the theoretical results obtained before. The generalized Adams-Bashforth-Moulton type predictor-corrector scheme is applied in order to find an approximate solution for our fractional-order system [59,60]. The role of prey's disease $(\beta)$, predator's attack rate $(\alpha)$, half saturation constant $(a)$, infected prey's death rate $\left(d_{2}\right)$ and fractional order $(q)$ are discussed to validate our fractional-order model (3). We consider the following set of parameters:

$$
\begin{aligned}
& r=11, \quad \beta=1, \quad d_{1}=1, \quad d_{2}=2.1, \quad d_{3}=0.5, \quad c=1, \\
& \gamma=0.04, \quad \alpha=1 \quad \text { and } \quad a=1,
\end{aligned}
$$

which were also used for the integer-order model [11]. For the parameter set (9), the condition $\Re_{0}=5.14019>1$ holds and we get the predator-extinction equilibrium point $E_{2}\left(S_{2}, I_{2}, 0\right)=(2.14,4.21905,0)$, also the conditions $\alpha=1>\frac{a d_{3}}{c}=0.5$ and $\Re_{0}=5.14019>$ $1+\frac{\beta d_{2} d_{3}}{d_{1}\left(\gamma+d_{2}\right)\left(c \alpha-a d_{3}\right)}=1.98131$ hold and one gets the interior equilibrium point $E_{3}\left(S_{3}, I_{3}, P_{3}\right)=$ $(5.52,1,6.76)$. The axial equilibrium point is $E_{1}\left(S_{1}, 0,0\right)=(11,0,0)$.

From Theorem 3, when $\beta=0.02$ and $q=0.9$, the condition of $\Re_{0}=0.102804<1$ holds and the axial equilibrium point $E_{1}(11,0,0)$ of the fractional-order system (3) is locally asymptotically stable as indicated in Fig. 1(b).

Following Theorem 4, when $\beta=0.24$ and $q=0.9$, the condition of $\Re_{0}=1.23364<1+$ $\frac{\beta d_{2} d_{3}}{d_{1}\left(\gamma+d_{2}\right)\left(c \alpha-a d_{3}\right)}=1.23551$ holds and the predator-extinction equilibrium point $E_{2}(8.91667$, $0.992063,0$ ) of the fractional-order system (3) is locally asymptotically stable as shown in Fig. 1(a).

One can observe that, the dynamics of the population is stable around the interior equilibrium point $E_{3}(5.52,1,6.76)$ in the presence of a prey's disease $(\beta=1)$ as shown in Fig. 2(a) coinciding with the bifurcation diagram 5 . Furthermore, the infected prey and predator population go to extinction in the absence of prey's disease $(\beta=0)$ as indicated 

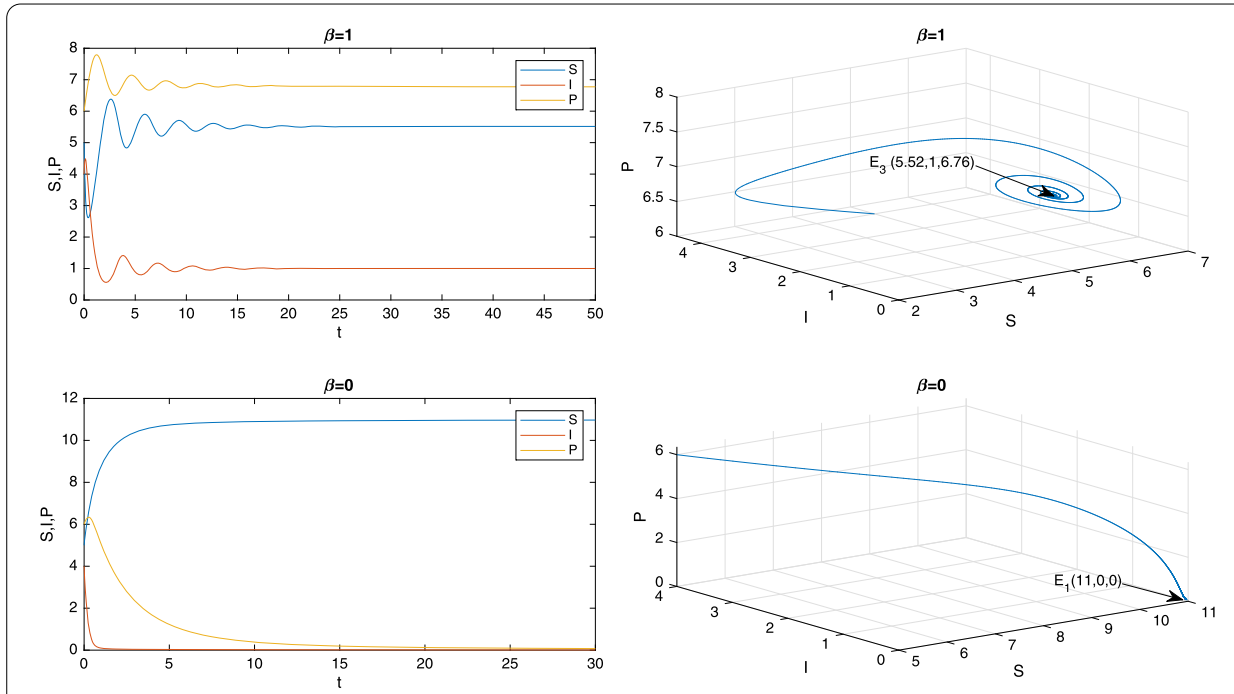

Figure 2 Time series and phase diagram of the stable interior equilibrium point $E_{3}(5.52,1,6.76)$ and axial equilibrium point $E_{1}(11,0,0)$ with different values of $\beta$ and $q=0.9$
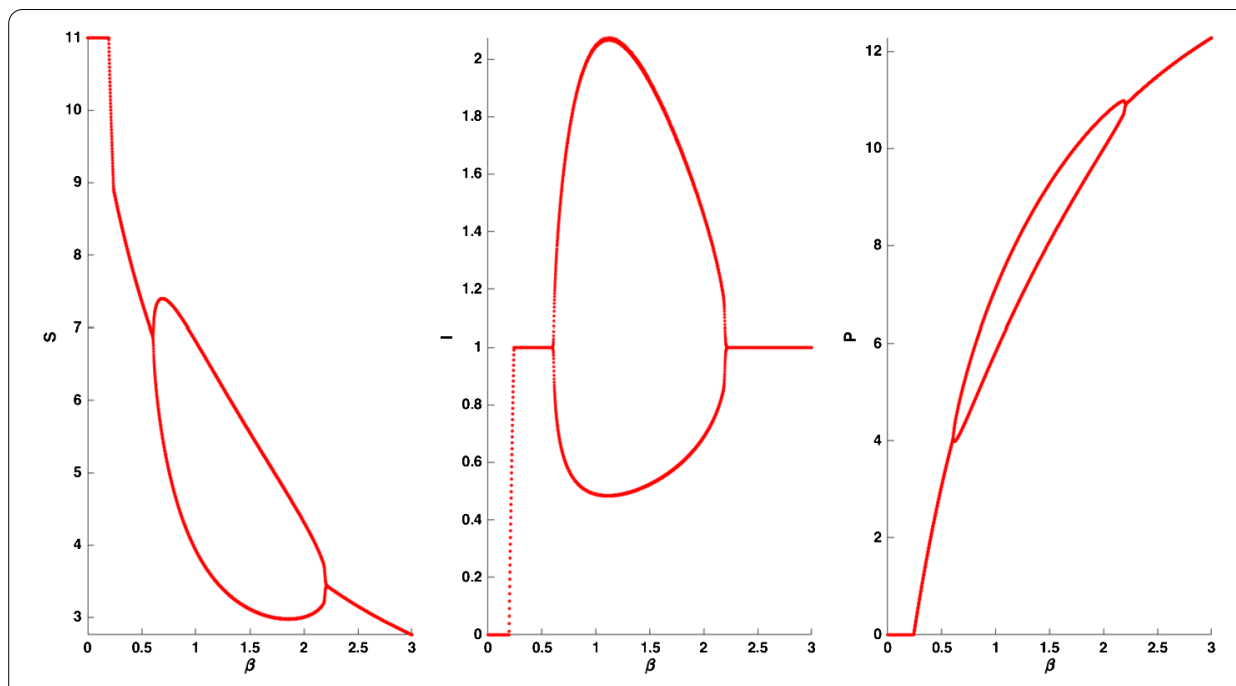

Figure 3 Bifurcation diagram of the fractional-order model (3) with respect to prey's disease $(\beta)$ when $q=1$

in Fig. 2(b) concurring with Fig. 5. Therefore, the prey's disease $(\beta)$ has a crucial role in population dynamics and may make the dynamics stable between populations.

For better visualization of the impact of $\beta$ on the dynamics of the integer-order ecoepidemiological model (when $q=1$ ), we draw the bifurcation diagram with respect to $\beta$ as shown in Fig. 3 coinciding with Fig. 4 and 6 . The transcritical bifurcations and supercritical Hopf bifurcations values localized at $\beta_{\mathrm{tr} 1}=0.194545, \beta_{\mathrm{tr} 2}=0.240449, \beta_{1}^{*}=0.608815$ and $\beta_{2}^{*}=2.19262$, respectively, are shown in Fig. 3. It is observed that the system (3) shows stable extinction of the infected prey and predator populations for $\beta<\beta_{\text {tr } 1}$, stable extinction of the predator population for $\beta_{\mathrm{tr} 1}<\beta<\beta_{\mathrm{tr} 2}$, stable coexistence of populations for $\beta_{\text {tr } 2}<\beta<\beta_{1}^{*}$, cyclic dynamics occur for $\beta_{1}^{*}<\beta<\beta_{2}^{*}$ and stable coexistence of populations for $\beta>\beta_{2}^{*}$ as shown in Fig. 3 coinciding with Figs. 4 and 6 . 


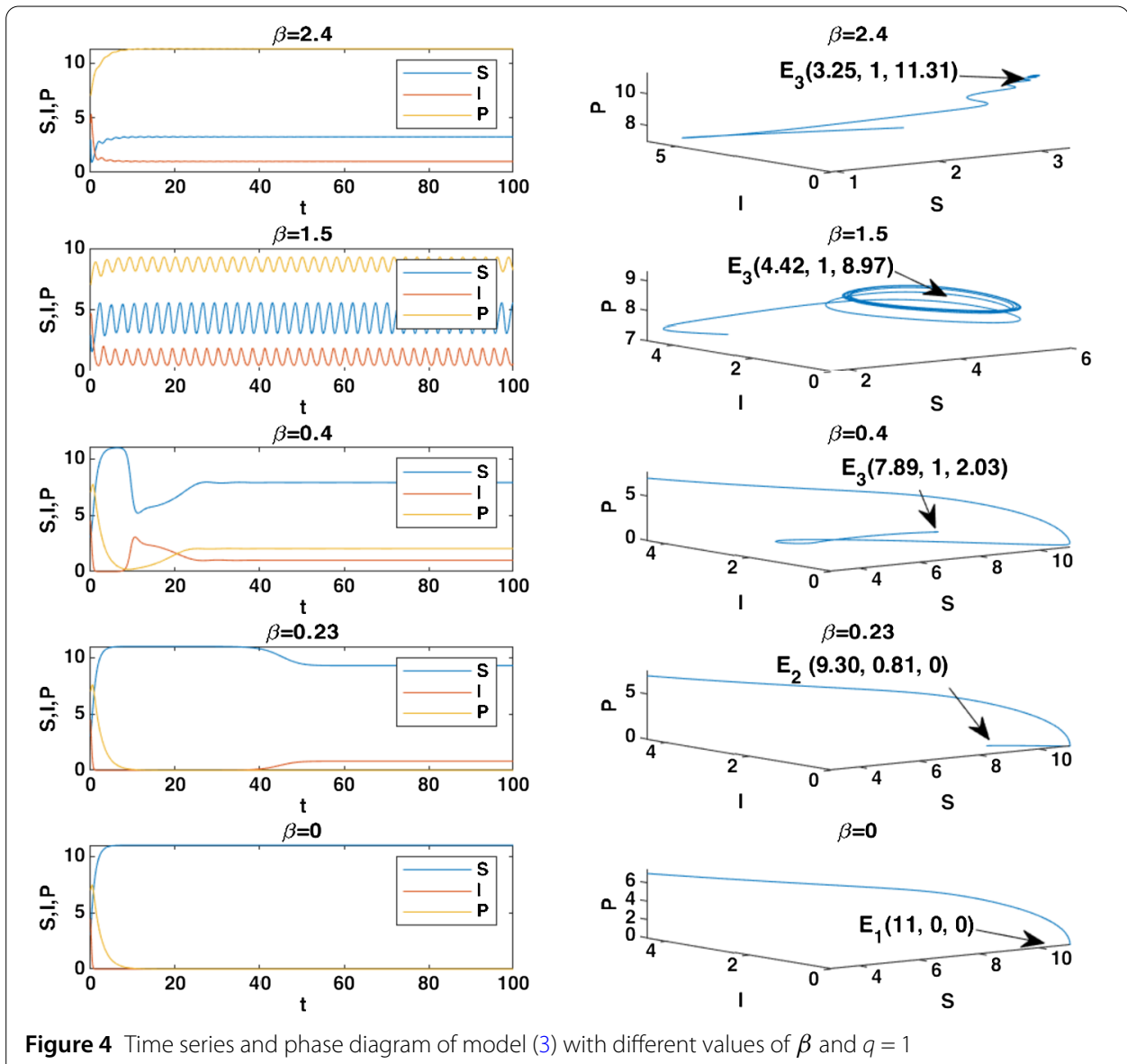

Figure 4 Time series and phase diagram of model (3) with different values of $\beta$ and $q=1$
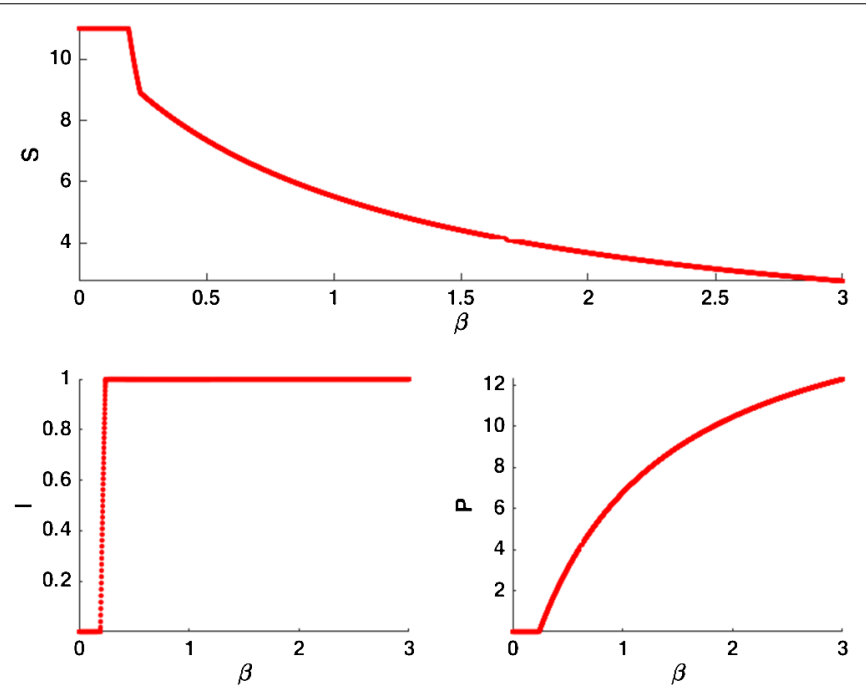

Figure 5 Bifurcation diagram of the fractional-order model (3) with respect to prey's disease $(\beta)$ when $q=0.95$

To depict the impact of prey's disease $(\beta)$ on the dynamics of the fractional-order ecoepidemiological model (3) (when $q=0.9$ ) the bifurcation diagram is depicted in Fig. 3 with respect to $\beta$. The transcritical bifurcation value localized at $\beta_{\mathrm{tr}}=0.240449$ is shown 


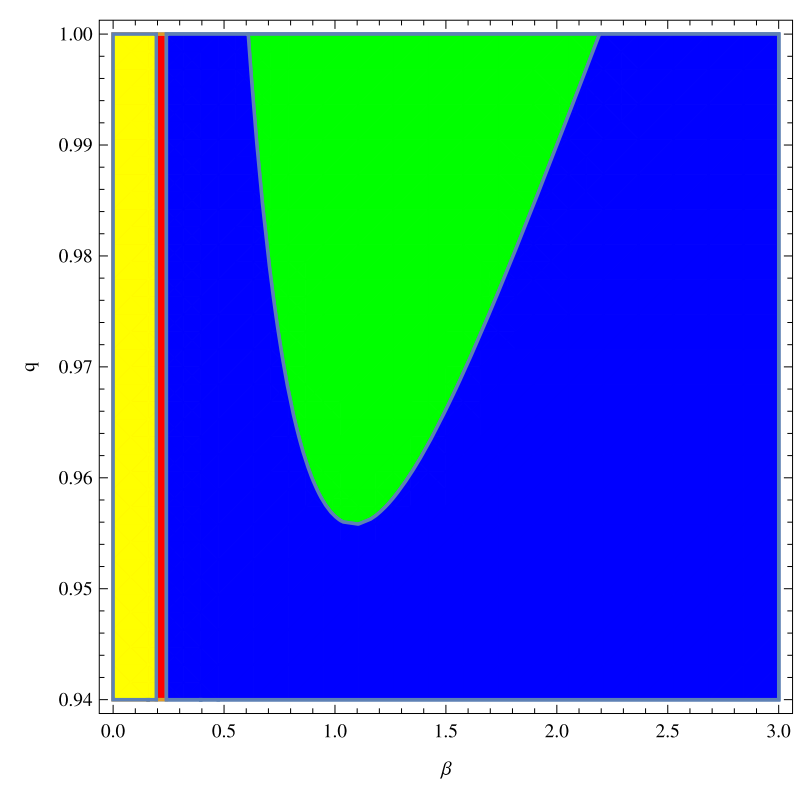

Figure 6 Stability regions of the fractional-order model (3) in the $(\beta-q)$ plane

in Fig. 5. From Fig. 3 it is observed that the integer-order system (when $q=1$ ) shows a limit cycle for $\beta_{1}^{*}<\beta<\beta_{2}^{*}$, which leads to the coexistence of populations in fractional-order case (when $q=0.95$ ) as shown in Fig. 5 coinciding with Fig. 6 . Therefore, the fractional order $(q)$ and prey's disease $(\beta)$ have stabilization effects and may help to control the disease.

Figure 6 shows the stability regions of the fractional-order system (3) with respect to the prey's disease $(\beta)$ and fractional order $(q)$. The regions are divided into four distinguished parts, where the yellow region shows the stable of axial equilibrium point $E_{1}$, the red region shows stability of the predator-extinction equilibrium point $E_{2}$, the blue region indicates the stability of the interior equilibrium point $E_{3}$ and the green region shows a limit cycle oscillation coinciding with theoretical results obtained before concurringring with Figs. 3 , 4 and 5. If we fix the parameter $\beta=1.5$, then for $q<0.966137$ the fractional-order system (3) is stable and the system changes its stability to show a limit cycle oscillation for $q>$ 0.966137 . It is to be noted that the three-species coexistence equilibrium point $E_{3}$ of the system (3) is stable in the fractional-order case when $q=0.95$ becomes unstable in the integer-order case when $q=1$. Therefore, the fractional order $(q)$ has stabilization effects and can be used to control the persistence of species in the ecosystem.

According to Theorem 6 one can observe that the condition of $\Re_{0}=0.1048<1$ holds when $\beta=0.02$, and the axial equilibrium point $E_{1}(11,0,0)$ of the fractional-order system (3) is globally asymptotically stable as indicated in Fig. 7.

Figure 8 indicates that all trajectories with different positive initial conditions converge to the equilibria with predator-extinction outcome, $E_{2}$ when $\beta=0.24$, which indicates that $E_{2}$ is globally asymptotically stable. In this case $\Re_{0}=1.23364<1+\frac{\beta d_{2} d_{3}}{d_{1}\left(\gamma+d_{2}\right)\left(c \alpha-a d_{3}\right)}=$ 1.23551, concurring with the results of Theorem 7 .

Figure 9 shows the bifurcation diagram around the interior equilibrium point $E_{3}$ with respect to the predator's attack rate $(\alpha)$ when $q=0.9$. It can be observed that the fractionalorder eco-epidemiological model (3) goes through the transcritical bifurcation value at $\alpha_{\mathrm{tr}}=0.61851$ and two supercritical Hopf bifurcation values at $\alpha_{1}^{*}=1.10378$ and $\alpha_{2}^{*}=$ 

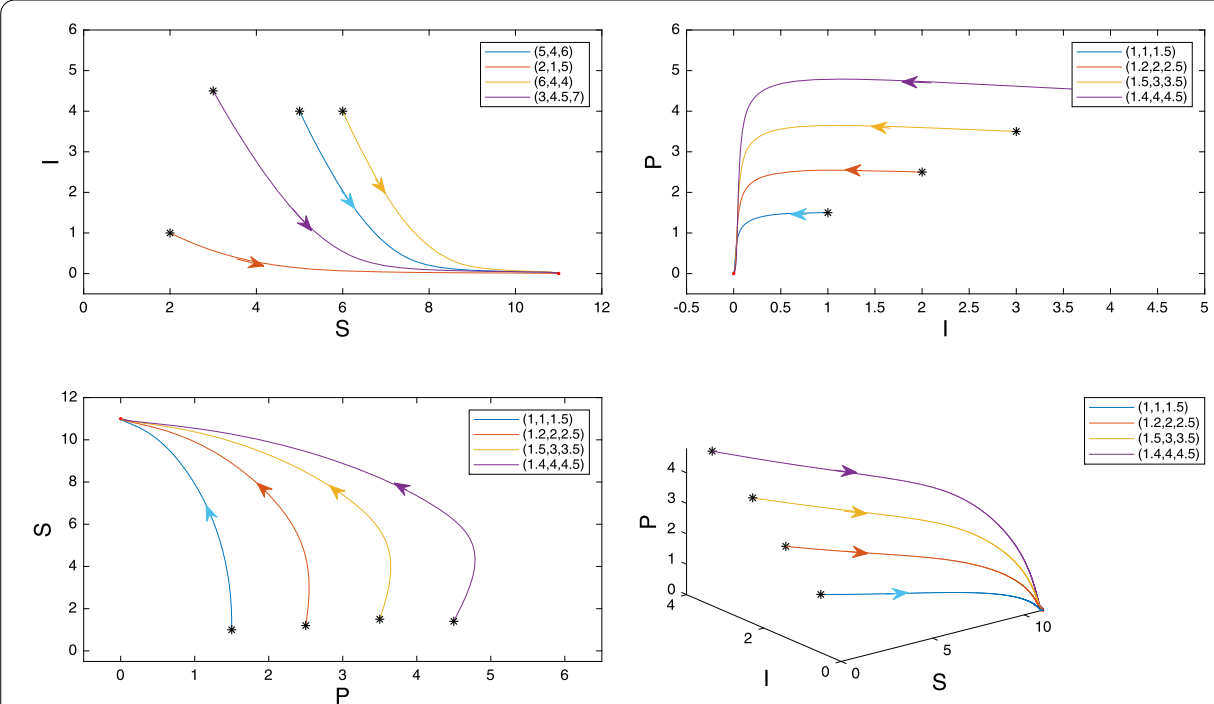

Figure 7 Global asymptotic stability of the axial equilibrium point $E_{1}$ with different initial values when $\beta=0.02$ and $q=0.9$
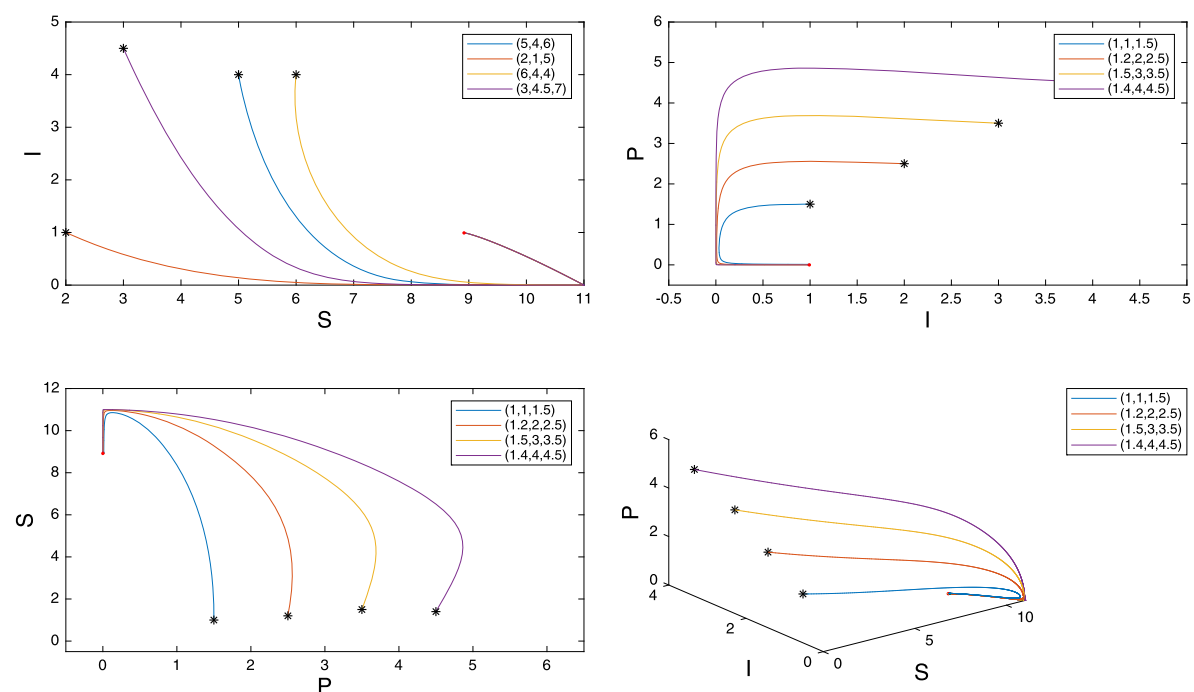

Figure $8 \mathrm{Global}$ asymptotic stability of the predator-extinction equilibrium point $E_{2}$ with different initial values when $\beta=0.24$ and $q=1$

4.3697, respectively, as shown in Fig. 9. One can observe that below the transcritical bifurcation value, the predator population goes extinct and the populations of susceptible prey and infected prey survive as depicted in Fig. 9 corresponding to Fig. 10 when $\alpha=0.25$. For the predator's attack rate $(\alpha)$ between the transcritical bifurcation value $\left(\alpha_{\mathrm{tr}}=0.61851\right)$ and supercritical Hopf bifurcation value $\left(\alpha_{1}^{*}=1.10378\right)$ the system depicts the stable coexistence of susceptible prey, infected prey and predator populations as shown in Fig. 9 concurring with Fig. 10 when $\alpha=0.99$. For the predator's attack rate $(\alpha)$ between supercritical Hopf bifurcation values $\left(\alpha_{1}^{*}=1.10378\right.$ and $\left.\alpha_{2}^{*}=4.3697\right)$ the system shows a limit cycle oscillation as exhibited in Fig. 9 concurring with Fig. 10 when $\alpha=1.5$ and $\alpha=4$. 

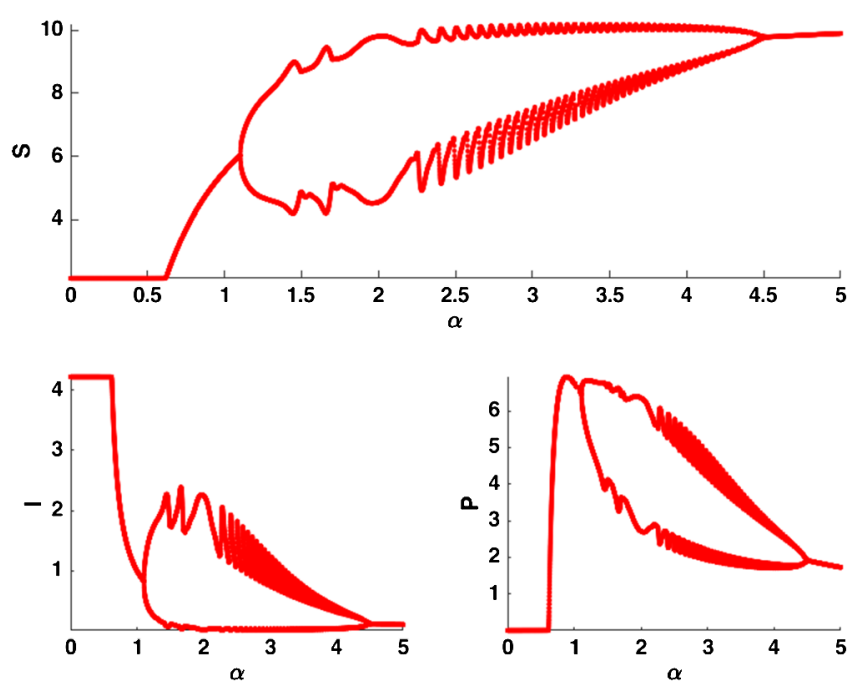

Figure 9 Bifurcation diagram of the fractional-order model (3) with respect to predator's attack rate $(\alpha)$ when $q=0.9$
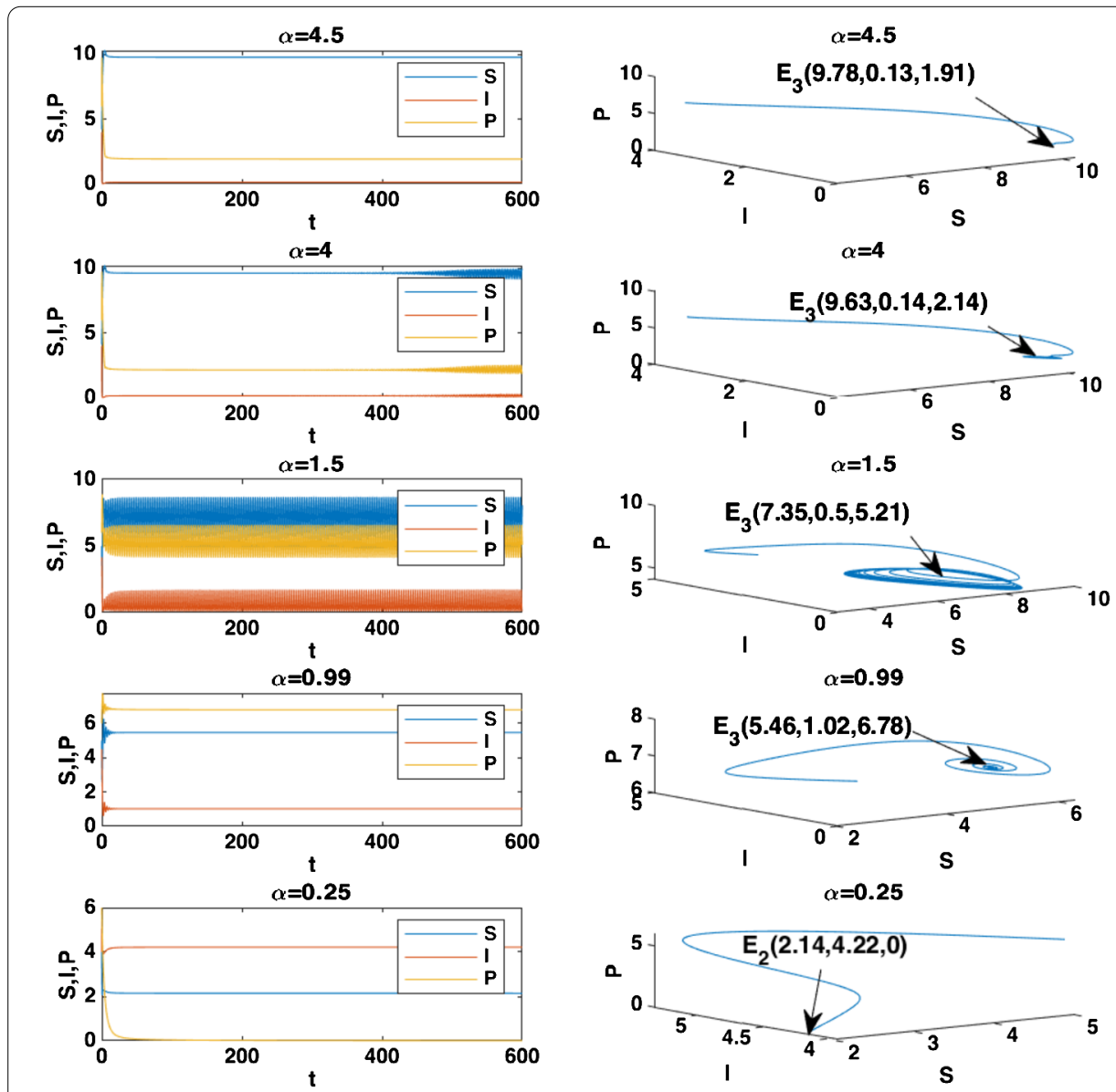

Figure 10 Time series and phase diagram of the fractional-order model (3) with different values of predator's attack rate $(\alpha)$ when $q=0.9$ 

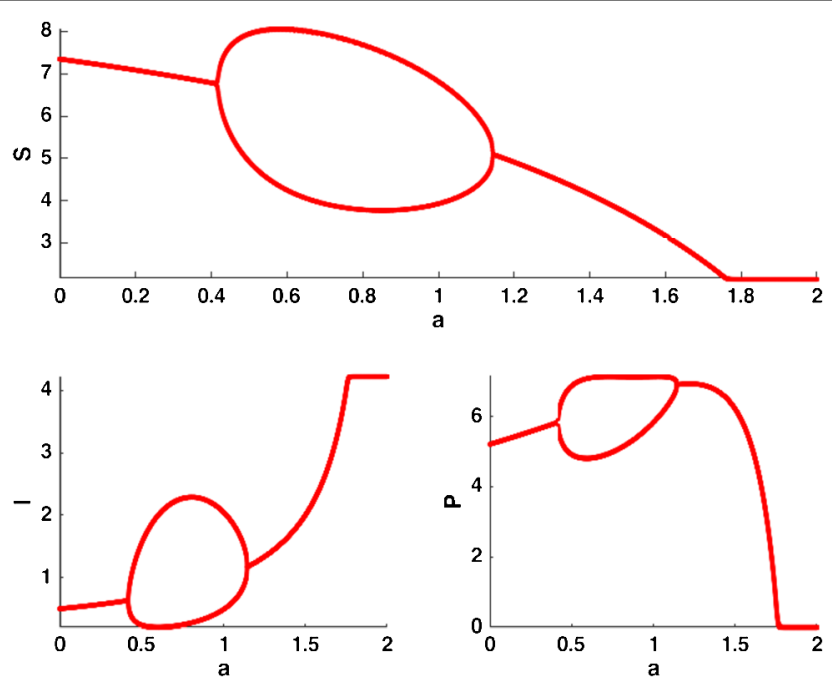

Figure 11 Bifurcation diagram of the fractional-order model (3) with respect to half saturation constant (a) when $q=1$
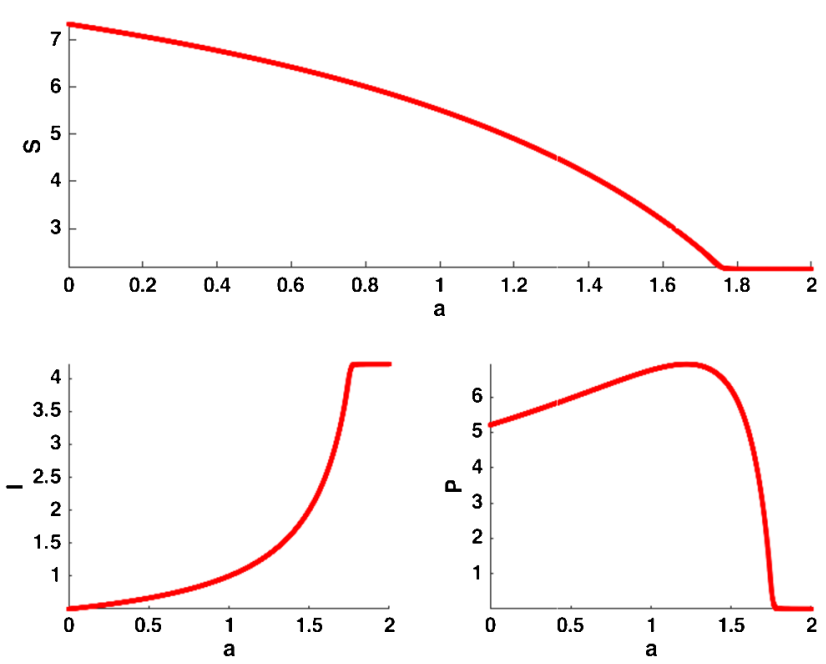

Figure 12 Bifurcation diagram of the fractional-order model (3) with respect to half saturation constant (a) when $q=0.9$

Above the supercritical Hopf bifurcation value $\left(\alpha_{2}^{*}=4.3697\right)$ the system exhibits the stable coexistence of populations as shown in Fig. 9 coinciding with Fig. 10 when $\alpha=4.5$. Therefore, one can control the coexistence of populations by setting the predator's attack rate between $\alpha_{\mathrm{tr}}=0.61851$ and $\alpha_{1}^{*}=1.10378$ or above the supercritical Hopf bifurcation value $\left(\alpha_{2}^{*}=4.3697\right)$. Note that the predator's attack rate $(\alpha)$ has a crucial effect on the dynamics of the fractional-order model (3) as shown in Fig. 9 coinciding with Fig. 10.

Figures 11 and 12 show the bifurcation analysis of the fractional-order system (3) with respect to half saturation constant $(a)$ for integer-order case (when $q=1$ ) and fractionalorder case (when $q=0.9$ ), respectively. Figure 11 shows that two Hopf bifurcation points and one transcritical bifurcation point emerge in the integer-order case (when $q=1$ ). On 


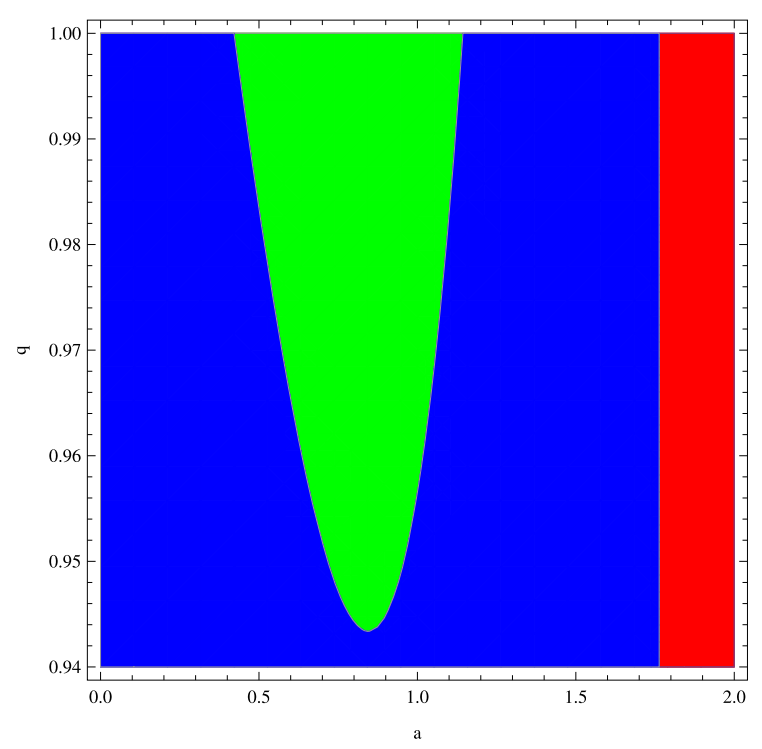

Figure 13 Stability regions of the fractional-order model (3) in ( $a-q)$ plane
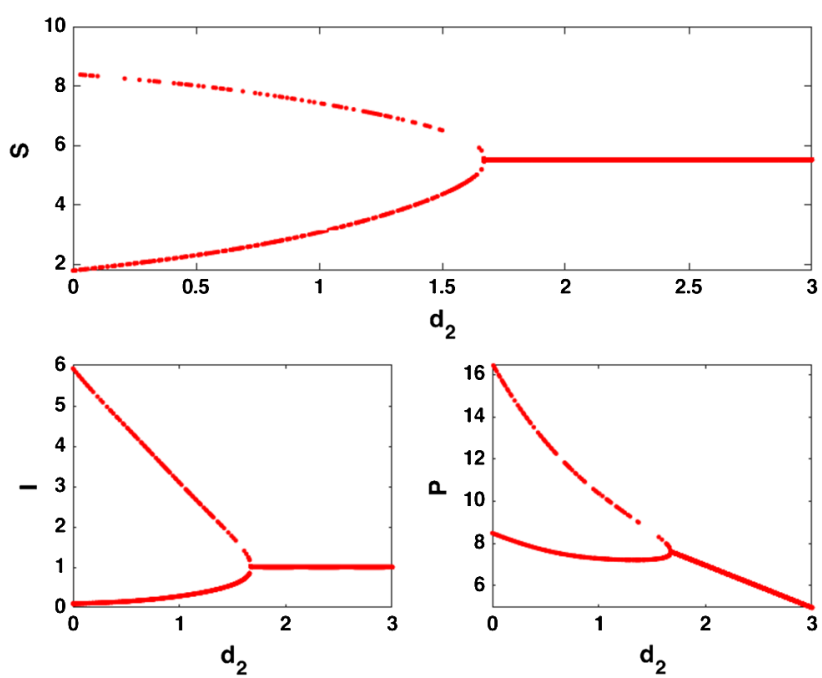

Figure 14 Bifurcation diagram of the fractional-order model (3) with respect to the infected prey's death rate $\left(d_{2}\right)$ when $q=0.9$

the other hand only one transcritical bifurcation point is observed in fractional-order case (when $q=0.9$ ) and this is clearly shown in Fig. 12 coinciding with Fig. 13.

The stability regions of the fractional-order system (3) with respect to the half saturation constant $(a)$ and fractional order $(q)$ are presented in Fig. 13. The blue region shows the stability of the interior equilibrium point $E_{3}$, the green region shows a limit cycle oscillation, the red region shows stability of predator-extinction equilibrium point $E_{2}$.

In order to show the impact of infected prey's death rate $\left(d_{2}\right)$ on the dynamics of the fractional-order eco-epidemiological model (3) the bifurcation diagram of the interior equilibrium point $E_{3}$ is drawn when $(q=0.9)$ as shown in Fig. 14. The supercritical Hopf 

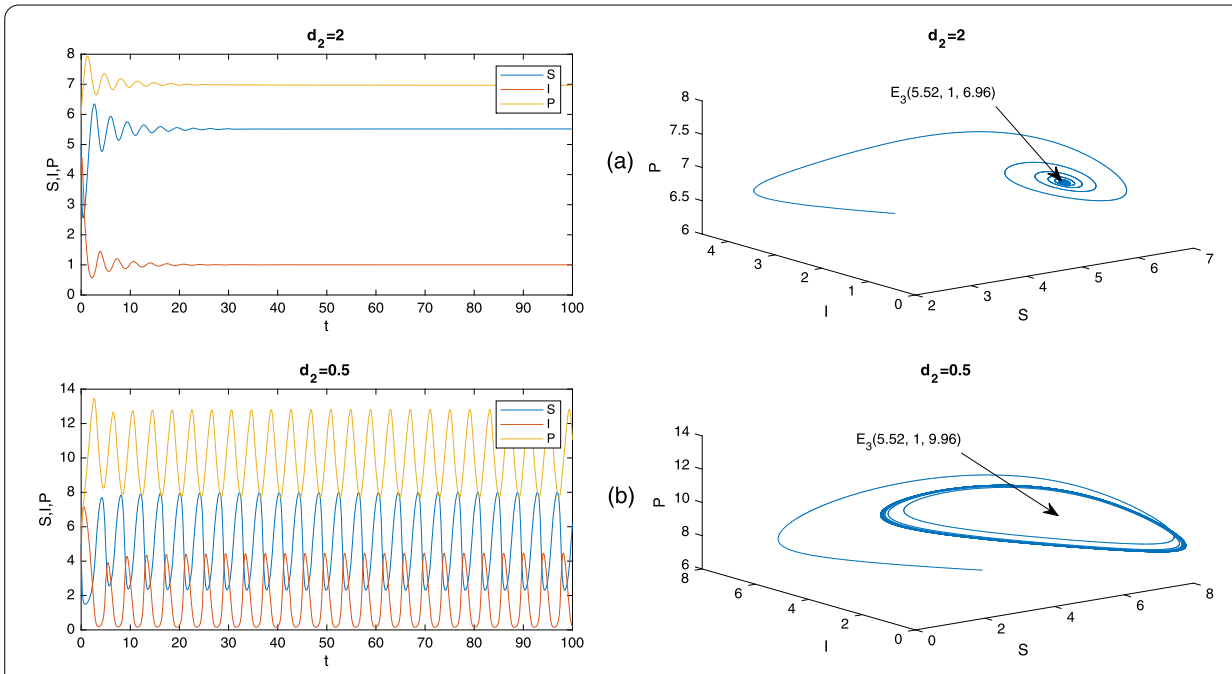

Figure 15 Time series and phase diagram of the fractional-order model (3) with different values of infected prey's death rate $\left(d_{2}\right)$ when $q=0.9$
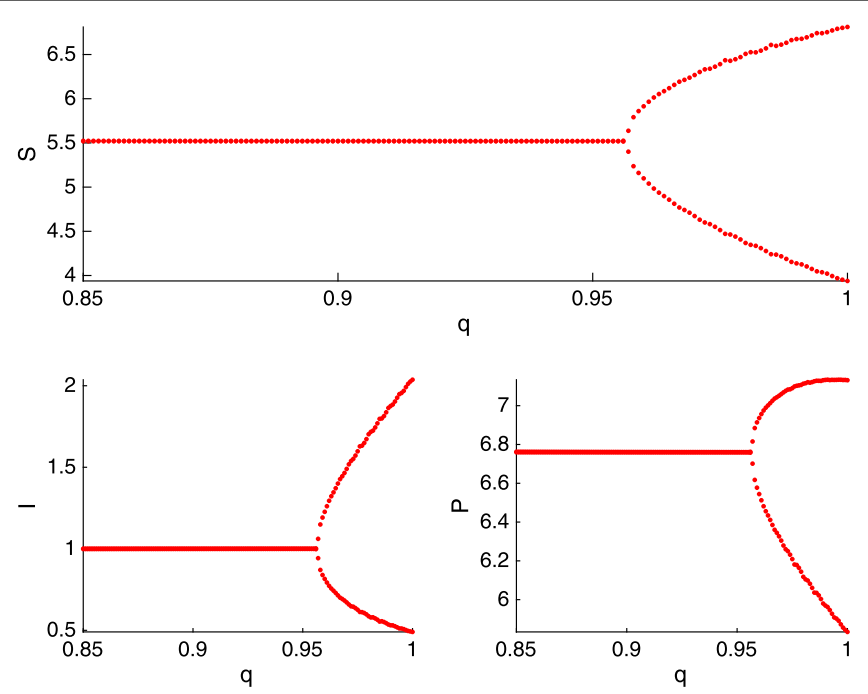

Figure 16 Bifurcation diagram of the fractional-order model (3) with respect to fractional order (q)

bifurcation value centralizes at $d_{2}^{*}=1.67897$ as indicated in Fig. 14 . When $d_{2}>d_{2}^{*}$, the interior equilibrium point $E_{3}$ is locally asymptotically stable as depicted in Fig. 14 concurring with Fig. 15(a) when $d_{2}=2$. For $d_{2}<d_{2}^{*}$, the system shows an unstable coexistence of susceptible prey, infected prey and predator populations as exhibited in Fig. 14 coinciding with Fig. 15(b) when $d_{2}=0.5$. Therefore, one can observe that the infected prey's death rate $\left(d_{2}\right)$ has a critical role in the dynamics of the fractional-order model (3).

For better understand the effects of fractional order $(q)$ on the dynamics of system (3) one can draw the bifurcation diagram considering fractional order $(q)$ as a bifurcation parameter. Figure 16 exhibits the bifurcation diagrams for susceptible prey, infected prey and predator populations with respect to $q$. The supercritical Hopf bifurcation value centralizes at $q^{*}=0.95653$ as indicated in Fig. 16. When $q<q^{*}$, the interior equilibrium 


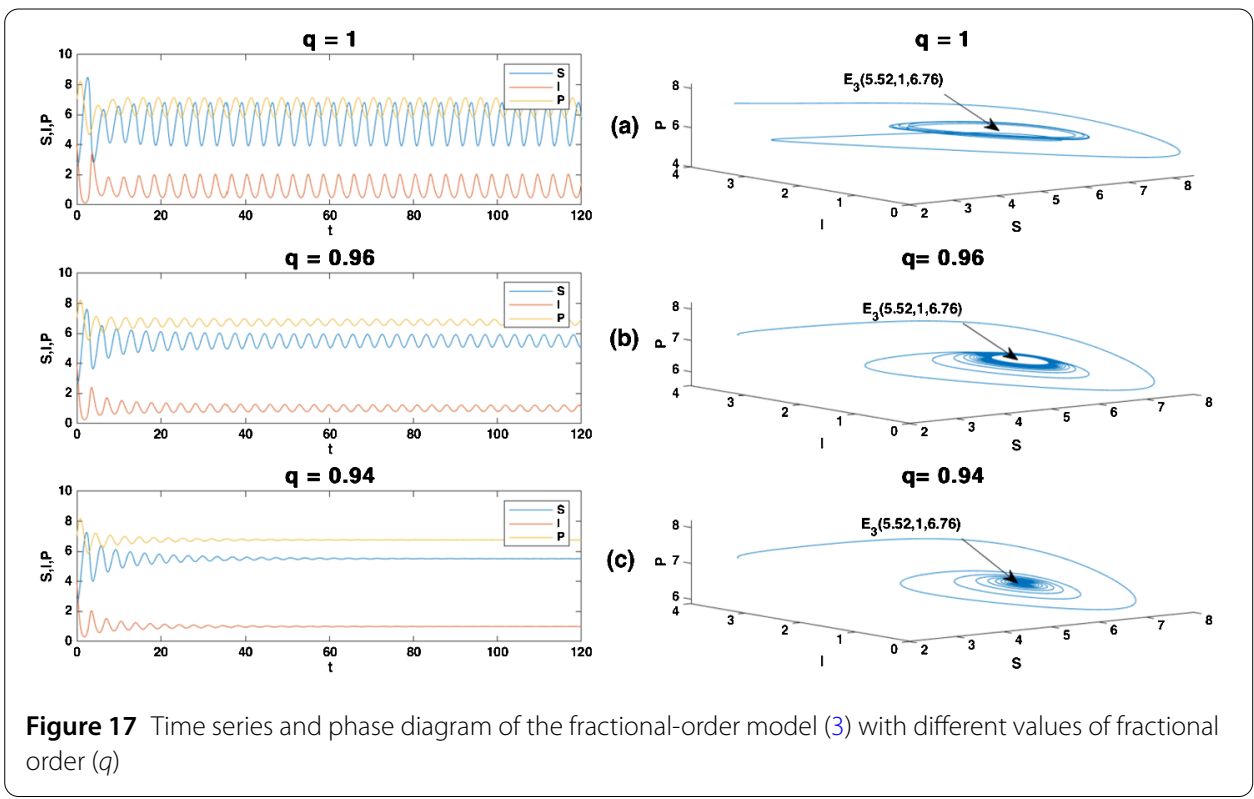

point $E_{3}(5.52,1,6.76)$ is locally asymptotically stable as shown in Fig. 16 concurring with Fig. 17 (c) when $q=0.94$. For $q>q^{*}$, the system undergoes limit cycle oscillations as exhibited in Fig. 16 coinciding with Fig. 17(b) when $q=0.96$. It is important to notice that when $q=1$ the fractional-order eco-epidemiological model (3) reduces to the epidemiological model [11]. Also, when $q=1$ and $\gamma=0$ the fractional-order eco-epidemiological model (3) reduces to the classical epidemiological model [10].

One can observe that the integer-order model (when $q=1$ ) shows periodic limit cycle dynamics as shown in Fig. 16 concurring with Fig. 17(a) when $q=1$. Furthermore, in the fractional-order case (when $q=0.94$ ) the system exhibits the stable co-existence of susceptible prey, infected prey and predator as indicated in Fig. 16 coinciding with Fig. 17(c) when $q=0.94$. Hence, the fractional order $(q)$ has stabilization effects and it may help to control the coexistence between of susceptible prey, infected prey and predator populations when $0<q<q^{*}$.

\section{Conclusion}

In this paper, a fractional-order eco-epidemiological model incorporating the predator's attack rate $(\alpha)$ and half saturation constant $(a)$ with infection in the prey population is formulated and analyzed in which the populations have been divided into susceptible prey, infected prey and predator. A sufficient condition for the existence as well as uniqueness of the fractional-order eco-epidemiological model (3) has been obtained. It has been proved that the solutions of the fractional-order system of differential equations (3) are uniformly bounded and non-negative. The local and global stability of the equilibrium points of the fractional-order eco-epidemiological model (3) has been investigated. The threshold parameter $\left(\Re_{0}\right)$ has been used to determine the existence and stability conditions of the equilibrium points. It has noted that the axial equilibrium point $E_{1}$ is locally asymptotically stable when the predator-extinction equilibrium point $E_{2}$ and the interior equilibrium point $E_{3}$ do not exist. Numerical simulations have been conducted to illustrate our analytical results. For better visualization of the effects of the prey's disease $(\beta)$, predator's attack rate $(\alpha)$, half saturation constant $(a)$, infected prey's death rate $\left(d_{2}\right)$ and fractional order $(q)$ on 
the dynamics of the fractional-order eco-epidemiological model (3) we have drawn the bifurcation diagrams with respect to $\beta, \alpha, a, d_{2}$ and $q$, respectively. It has been shown that the prey's disease may stabilize the system. Also, it has to be noted the predator's attack rate, the half saturation constant and the infected prey's death rate have crucial effect on the dynamics of the fractional-order model (3). Furthermore, it is observed that the fractional order $(q)$ is important for the stabilization of the system and it may help to control the coexistence between of susceptible prey, infected prey and predator populations when $0<q<q^{*}$.

\author{
Acknowledgements \\ The authors would like to express their sincere thanks to the editor and the anonymous reviewers for their helpful \\ comments and suggestions.
}

\title{
Funding
}

This work was supported by the Fundamental Research Grant Scheme (Sponsor-Ministry of Education Malaysia (MOE) Acct No: 203/PMATHS/6711570), Bridging Grant scheme (Sponsor: Research Creativity and Management Office (RCMO), Universiti Sains Malaysia, Acct no: 304/PMATHS/6316285).

\section{Availability of data and materials}

Not applicable.

\section{Competing interests}

The authors declare that they have no competing interests.

Authors' contributions

All authors read and approved the final manuscript.

\section{Publisher's Note}

Springer Nature remains neutral with regard to jurisdictional claims in published maps and institutional affiliations.

Received: 9 May 2019 Accepted: 16 January 2020 Published online: 23 January 2020

\section{References}

1. Boccara, N.: Modeling Complex Systems. Springer, Berlin (2010)

2. Khajanchi, S.: Modeling the dynamics of stage-structure predator-prey system with Monod-Haldane type response function. Appl. Math. Comput. 302, 122-143 (2017)

3. Nosrati, K., Shafiee, M.: Dynamic analysis of fractional-order singular Holling type-Il predator-prey system. Appl. Math. Comput. 313, 159-179 (2017)

4. Zhang, F., Chen, Y., Li, J.: Dynamical analysis of a stage-structured predator-prey model with cannibalism. Math. Biosci. 307, 33-41 (2019)

5. Moustafa, M., Mohd, M.H., Ismail, A.I., Abdullah, F.A.: Stage structure and refuge effects in the dynamical analysis of a fractional order Rosenzweig-MacArthur prey-predator model. Prog. Fract. Differ. Appl. 5, 1-16 (2019)

6. Kermack, W., Mckendrick, A.: A contribution to the mathematical theory of epidemics. Proc. R. Soc. A 115, 700-721 (1927)

7. Li, H., Zhang, L., Teng, Z., Jiang, Y., Muhammadhaji, A.: Global stability of an SI epidemic model with feedback controls in a patchy environment. Appl. Math. Comput. 321, 372-384 (2018)

8. Anderson, R.M., May, R.M.: Infectious Diseases of Humans: Dynamics and Control. Oxford University Press, London (1992)

9. Stone, L., Shulgin, B., Agur, Z.: Theoretical examination of the pulse vaccination policy in the SIR epidemic model. Math. Comput. Model. 31, 207-215 (2000)

10. Mukherjee, D.: Hopf bifurcation in an eco-epidemic model. Appl. Math. Comput. 217, $2118-2124$ (2010)

11. Juneja, N., Agnihotri, K.: Conservation of a predator species in SIS prey-predator system using optimal taxation policy. Chaos Solitons Fractals 116, 86-94 (2018)

12. Hilker, F.M., Schmitz, K.: Disease-induced stabilization of predator-prey oscillations. J. Theor. Biol. 255, 299-306 (2008)

13. Mortoja, G., Panja, P., Mondal, K.: Dynamics of a predator-prey model with nonlinear incidence rate, Crowley-Martin type functional response and disease in prey population. Ecol. Genet. Gen. 10, 100035 (2018)

14. Meng, X., Qin, N., Huo, H.: Dynamics analysis of a predator-prey system with harvesting prey and disease in prey species. J. Biol. Dyn. 12, 342-374 (2018)

15. Greenhalgh, D., Haque, M.: A predator-prey model with disease in the prey species only. Math. Methods Appl. Sci. 30, 911-929 (2007)

16. Shaikh, A.A., Das, H., Sarwardi, S.: Dynamics of an eco-epidemiological system with disease in competitive prey species. J. Appl. Math. Comput. (2019). https://doi.org/10.1007/s12190-019-01295-6

17. Rana, S., Samanta, S., Bhattacharya, S.: The interplay of Allee effect in an eco-epidemiological system with disease in predator population. Bull. Calcutta Math. Soc. 108, 103-122 (2016)

18. Juneja, N., Agnihotri, K.: Global stability of harvested prey-predator model with infection in predator species. In: Information and Decision Sciences, pp. 559-568. Springer, Berlin (2018) 
19. Pal, P.J., Haque, M., Mandal, P.K.: Dynamics of a predator-prey model with disease in the predator. Math. Methods Appl. Sci. 37, 2429-2450 (2014)

20. Bulai, I.M., Hilker, F.M.: Eco-epidemiological interactions with predator interference and infection. Theor. Popul. Biol. 130, 191-202 (2019)

21. Agnihotri, K., Juneja, N.: An eco-epidemic model with disease in both prey and predator. IJAEEE 4, 50-54 (2015)

22. Hsieh, Y., Hsiao, C.: Predator-prey model with disease infection in both populations. Math. Med. Biol. 25, 247-266 (2008)

23. Gao, X., Pan, Q., He, M., Kang, Y.: A predator-prey model with diseases in both prey and predator. Phys. A, Stat. Mech. Appl. 392, 5898-5906 (2013)

24. Almeida, R., Cruz, B., Martins, N., Monteiro, T.: An epidemiological MSEIR model described by the Caputo fractional derivative. Int. J. Dyn. Control 7, 776-784 (2018)

25. Heymans, N., Podlubny, I.: Physical interpretation of initial conditions for fractional differential equations with Riemann-Liouville fractional derivatives. Rheol. Acta 45, 765-771 (2006)

26. Kilbas, A.A., Srivastava, H.M.. Trujillo, J.J.: Theory and Applications of Fractional Differential Equations. Elsevier, Amsterdam (2006)

27. Ansari, S.P., Agrawal, S.K., Das, S.: Stability analysis of fractional-order generalized chaotic susceptible-infected-recovered epidemic model and its synchronization using active control method. Pramana 84 23-32 (2015)

28. Santos, J.P.C., Cardoso, L.C., Monteiro, E., Lemes, N.: A fractional-order epidemic model for bovine babesiosis disease and tick populations. In: Abstract and Applied Analysis, Hindawi, vol. 2015 (2015)

29. Zhao, D., Luo, M.: Representations of acting processes and memory effects: general fractional derivative and its application to theory of heat conduction with finite wave speeds. Appl. Math. Comput. 346, 531-544 (2019)

30. Bolton, L., Cloot, A.H., Schoombie, S.W., Slabbert, J.P.: A proposed fractional-order Gompertz model and its application to tumour growth data. Math. Med. Biol. 32, 187-207 (2014)

31. Li, H., Muhammadhaji, A., Zhang, L., Teng, Z.: Stability analysis of a fractional-order predator-prey model incorporating a constant prey refuge and feedback control. Adv. Differ. Equ. 2018, 325 (2018)

32. Ahmed, E., El-Sayed, A., El-Saka, H.A.: Equilibrium points, stability and numerical solutions of fractional-order predator-prey and rabies models. J. Math. Anal. Appl. 325, 542-553 (2007)

33. Mondal, S., Lahiri, A., Bairagi, N.: Analysis of a fractional order eco-epidemiological model with prey infection and type 2 functional response. Math. Methods Appl. Sci. 40, 6776-6789 (2017)

34. Nugraheni, K., Trisilowati, T., Suryanto, A.: Dynamics of a fractional order eco-epidemiological model. J. Trop. Life Sci. 7, 243-250 (2017)

35. Delavari, H., Baleanu, D., Sadati, J.: Stability analysis of Caputo fractional-order nonlinear systems revisited. Nonlinear Dyn. 67, 2433-2439 (2012)

36. Moustafa, M., Mohd, M.H., Ismail, A.l., Abdullah, F.A.: Dynamical analysis of a fractional-order Rosenzweig-MacArthur model incorporating a prey refuge. Chaos Solitons Fractals 109, 1-13 (2018)

37. Matouk, A.E., Elsadany, A.A.: Dynamical analysis, stabilization and discretization of a chaotic fractional-order GLV model. Nonlinear Dyn. 85, 1597-1612 (2016)

38. Javidi, M., Ahmad, B.: Dynamic analysis of time fractional order phytoplankton-toxic phytoplankton-zooplankton system. Ecol. Model. 318, 8-18 (2015)

39. Sambath, M., Ramesh, P., Balachandran, K.: Asymptotic behavior of the fractional order three species prey-predator model. Int. J. Nonlinear Sci. Numer. Simul. 19, 721-733 (2018)

40. Suryanto, A., Darti, I., Panigoro, H.S., Kilicman, A.: A fractional-order predator-prey model with ratio-dependent functional response and linear harvesting. Mathematics 7, 1100 (2019)

41. Wang, X., Wang, Z., Xia, J.: Stability and bifurcation control of a delayed fractional-order eco-epidemiological model with incommensurate orders. J. Franklin Inst. 356, 8278-8295 (2019)

42. Area, I., Losada, J., Nieto, J.J.: On fractional derivatives and primitives of periodic functions. In: Abstract and Applied Analysis, Hindawi, vol. 2014 (2014)

43. Kaslik, E., Sivasundaram, S.: Non-existence of periodic solutions in fractional-order dynamical systems and a remarkable difference between integer and fractional-order derivatives of periodic functions. Nonlinear Anal., Real World Appl. 13, 1489-1497 (2012)

44. Li, X., Wu, R.: Hopf bifurcation analysis of a new commensurate fractional-order hyperchaotic system. Nonlinear Dyn 78, 279-288 (2014)

45. Yuan, L.G., Kuang, J.H.: Stability and a numerical solution of fractional-order Brusselator chemical reaction system. J. Fract. Calc. Appl. 8, 38-47 (2017)

46. González-Parra, G., Arenas, A.J., Chen-Charpentier, B.M.: A fractional order epidemic model for the simulation of outbreaks of influenza A (H1N1). Math. Methods Appl. Sci. 37, 2218-2226 (2014)

47. Diethelm, K.: A fractional calculus based model for the simulation of an outbreak of dengue fever. Nonlinear Dyn. 71(4), 613-619 (2013)

48. Arenas, A.J., González-Parra, G., Chen-Charpentier, B.M.: Construction of nonstandard finite difference schemes for the SI and SIR epidemic models of fractional order. Math. Comput. Simul. 121, 48-63 (2016)

49. Das, M., Maiti, A., Samanta, G.P.: Stability analysis of a prey-predator fractional order model incorporating prey refuge. Ecol. Genet. Gen. 7, 33-46 (2018)

50. Li, H., Zhang, L., Hu, C., Jiang, Y., Teng, Z.: Dynamical analysis of a fractional-order predator-prey model incorporating a prey refuge. J. Appl. Math. Comput. 54, 435-449 (2016)

51. Boukhouima, A., Hattaf, K., Yousfi, N.: Dynamics of a fractional order HIV infection model with specific functional response and cure rate. Int. J. Differ. Equ. 2017, Article ID 8372140 (2017)

52. Choi, S.K., Kang, B., Koo, N.: Stability for Caputo fractional differential systems. Abstr. Appl. Anal. 2014, Article ID 631419 (2014)

53. Driessche, P., Watmough, J.: Reproduction numbers and sub-threshold endemic equilibria for compartmental models of disease transmission. Math. Biosci. 180, 29-48 (2002)

54. Petras, l.: Fractional-Order Nonlinear Systems: Modeling, Analysis and Simulation. Springer, Berlin (2011) 
55. Matouk, A.E.: Chaos, feedback control and synchronization of a fractional-order modified autonomous Van der Pol-Duffing circuit. Commun. Nonlinear Sci. Numer. Simul. 16, 975-986 (2011)

56. Abdelouahab, M.S., Hamri, N., Wang, J.: Hopf bifurcation and chaos in fractional-order modified hybrid optical system Nonlinear Dyn. 69, 275-284 (2012)

57. Vargas-De-León, C.: Volterra-type Lyapunov functions for fractional-order epidemic systems. Commun. Nonlinear Sci. Numer. Simul. 24, 75-85 (2015)

58. Huo, J., Zhao, H., Zhu, L.: The effect of vaccines on backward bifurcation in a fractional order HIV model. Nonlinear Anal., Real World Appl. 26, 289-305 (2015)

59. Diethelm, K., Ford, N.J., Freed, A.D.: A predictor-corrector approach for the numerical solution of fractional differential equations. Nonlinear Dyn. 29, 3-22 (2002)

60. Li, C., Tao, C.: On the fractional Adams method. Comput. Math. Appl. 58, 1573-1588 (2009)

Submit your manuscript to a SpringerOpen ${ }^{\circ}$ journal and benefit from:

- Convenient online submission

- Rigorous peer review

- Open access: articles freely available online

- High visibility within the field

- Retaining the copyright to your article

Submit your next manuscript at $\boldsymbol{~ s p r i n g e r o p e n . c o m ~}$ 